\title{
Non-Markovian data-driven modeling of single-cell motility
}

\author{
Bernhard G. Mitterwallner $\odot$, Christoph Schreiber, Jan O. Daldrop, Joachim O. Rädler $\odot$, and Roland R. Netz ${ }^{*}$ \\ Fachbereich Physik, Freie Universität Berlin, 14195 Berlin, Germany and Physik Fakultät, Ludwig Maximilians Universität, \\ 80539 München, Germany
}

(Received 8 October 2019; accepted 7 January 2020; published 12 March 2020)

\begin{abstract}
Trajectories of human breast cancer cells moving on one-dimensional circular tracks are modeled by the non-Markovian version of the Langevin equation that includes an arbitrary memory function. When averaged over cells, the velocity distribution exhibits spurious non-Gaussian behavior, while single cells are characterized by Gaussian velocity distributions. Accordingly, the data are described by a linear memory model which includes different random walk models that were previously used to account for various aspects of cell motility such as migratory persistence, non-Markovian effects, colored noise, and anomalous diffusion. The memory function is extracted from the trajectory data without restrictions or assumptions, thus making our approach truly data driven, and is used for unbiased single-cell comparison. The cell memory displays time-delayed single-exponential negative friction, which clearly distinguishes cell motion from the simple persistent random walk model and suggests a regulatory feedback mechanism that controls cell migration. Based on the extracted memory function we formulate a generalized exactly solvable cell migration model which indicates that negative friction generates cell persistence over long timescales. The nonequilibrium character of cell motion is investigated by mapping the non-Markovian Langevin equation with memory onto a Markovian model that involves a hidden degree of freedom and is equivalent to the underdamped active Ornstein-Uhlenbeck process.
\end{abstract}

DOI: 10.1103/PhysRevE.101.032408

\section{INTRODUCTION}

The motion of single eukaryotic cells and bacteria is important for their survival and functioning and constitutes a characteristic phenotype. Also in multicellular organisms, single-cell motion is relevant for immunogenic response, embryonic development, and cancer metastasis [1-4]. The quantitative modeling of cell motility has progressed hand in hand with experimental techniques that allow for the automated tracking of single cells in different environments. The persistent random walk (PRW) model is conceptually simple, analytically solvable, and has been shown to account for many characteristic features of the motion of cells, bacteria, and entire organisms, in particular the crossover from ballistic motion at short times to diffusive motion at long times [5-8]. With increasing data quality, deviations from the simple PRW behavior have moved into the focus [9-13].

The PRW model is described by a linear Langevin equation with Gaussian white noise and thus implies Gaussian distributed velocities. However, cell migration data typically exhibit non-Gaussian velocity or step size distributions, which suggests the presence of nonlinearities. Nonlinear terms in the Langevin equation can reside in the deterministic or in the stochastic part, the latter is referred to as multiplicative noise, and have been shown to account for various motility properties of living matter [14-16]. An alternative explanation for non-Gaussian distributions was recently provided by single-cell motility analysis: while individual cell data were shown to be Gaussian, the significant deviations of the velocity distributions from cell to cell were demonstrated

*rnetz@physik.fu-berlin.de to lead to non-Gaussian behavior of the average over cells [17]. Since most motility analyses are based on averages over many cells or organisms, it is therefore not clear whether the often observed non-Gaussian behavior of averages is due to cell-to-cell variations or due to intrinsic nonlinear single-cell properties.

Aside from being linear, the PRW model predicts a velocity autocorrelation function (VACF) that decays single exponentially in time. In many experiments, multiexponential or even power-law decay of the VACF has been seen [18,19], which hints to memory and colored noise effects. On the other hand, multiexponential time correlations can also be caused by nonlinearities in the stochastic model. So, the situation is quite complex since nonlinearities in the stochastic equations and non-Markovian (i.e., colored-noise) effects can lead to similar signatures in the resulting trajectories. The analysis of a general model that would include all these effects is analytically not possible and necessarily involves numerical techniques and a large number of different parameters. The main problem, however, is that experimental data typically do not allow for a unique determination of model parameters since the organism lifetime and the sampling rate are limited and also since properties of individual cells vary in a given population. An additional complication is produced by the fact that living cells and organisms are nonequilibrium objects and thus fundamental statistical mechanics theorems, such as the fluctuation-dissipation relation, do not apply [20]. This means that in the description of motility data in terms of stochastic differential equations, even more parameters are present than for an equilibrium system.

What is direly needed is a data-based, unbiased analysis of experimental motility data which is able to deal with all the above mentioned effects. Here, we introduce such a method, 
which is based on the generalized Langevin equation (GLE) [21-23]. The GLE has found ample applications in the modeling of molecular systems [24-26], microrheological data $[27,28]$, and of non-Markovian dynamics along generalized reaction coordinates [26,29-33]. The GLE includes colored noise and accounts for non-Markovian effects by a memory kernel that describes for how long the system remembers its past states. We extract the memory kernel from cell trajectory data without prior assumptions on the functional form of the memory function, and compare the memory functions on the single-cell level. The Gaussian character of the single-cell velocity distributions allows us to use the linear Langevin equation, but we note that our memory-extraction method in principle also works for nonlinear systems [26].

Our study employs one-dimensional cell migration on microcontact printed tracks $[34,35]$. Specifically, we analyze a large data set of human breast cancer cells on homogeneous circular tracks, which is a setup we previously introduced and which has the advantage that long, one-dimensional time traces of many cells can be monitored without mutual interference [36]. The single-cell analysis reveals stationary statistics as well as almost perfect Gaussian velocity distributions with significant cell-to-cell variations. This means that inhomogeneous effects in time (i.e., time-dependent variations of cell properties) as well as multiplicative noise effects are absent. The VACF data exhibit significant nonexponential decay with time, which in the absence of non-Gaussian statistics is unambiguously traced back to non-Markovian memory effects. We extract the memory function from single-cell data as well as from data that are averaged over all cells, the good qualitative agreement of the resulting memory functions demonstrates that the main non-Markovian features are robust even in the presence of significant cell-to-cell variability. The memory function consists of an instantaneous friction contribution and a negative single-exponential part which decays over a few minutes. The GLE for such a memory kernel can be solved analytically, which allows us to investigate the migratory behavior of a general class of non-Markovian cell models. We find that negative exponentially decaying friction generates cell persistence on long timescales. Our correlation analysis of single-cell migratory parameters such as cell speed, cell diffusivity, and cell persistence reveals large spreading and only weak correlations, in particular, the cells do not partition into separate clusters. In contrast, strong correlations between memory function parameters are present, which gives rise to enhanced migratory persistence. We also consider effects due to finite-time discretization and due to localization noise, and show that our memory extraction methods are robust with respect to these two omnipresent experimental effects.

By an exact mapping, we demonstrate that the nonMarkovian memory function we extract from the cell data can be obtained from different Markovian equilibrium and nonequilibrium two-dimensional stochastic models in the presence of white noise. As we explain in detail, this mapping is not unique, meaning that there are many different Markovian models that give rise to the same non-Markovian dynamics. Nevertheless, such a mapping allows to investigate possible biochemical reaction networks that might underlie the observed migratory dynamics and to characterize classes of equivalent Markovian models. By this, our approach goes considerably beyond the mere fitting of model predictions to experimental data. We anticipate future applications of our methods to time series data from a broad class of different active systems.

\section{RESULTS AND DISCUSSION}

\section{A. Experimental trajectories}

MDA-MB-436 human breast cancer cells are confined to circular microlanes of mean radius $r_{0}=50 \mu \mathrm{m}$ and width $20 \mu \mathrm{m}$ [see inset of Fig. 1(a)]. This mesenchymal cell line serves as a model system for cells with robust motility. Circular microlanes have the advantage that cells do not move out of the field of view, which enables us to generate long trajectories of single cells with a duration that is limited only by the division time of the cells. The effect of lane curvature on cell migratory behavior was recently studied and found to be moderate [37]. The width of the microlane is chosen to be slightly smaller than the cell diameter, which produces quasi-one-dimensional motion of the confined cells. The cell position is determined as the center of fluorescence of the labeled cell nuclei. As a tradeoff between good statistics and limited photostress on the cell, fluorescence images of many different cells are recorded in parallel every $\Delta=2.5 \mathrm{~min}$ (see Appendix A for further details). Figure 1(a) shows example trajectories $x(t)=r_{0} \varphi(t)$, where $\varphi(t)$ is the angular position of the cell nucleus. We record $N=125$ trajectories up to a maximal time of $T=2155 \mathrm{~min}$, the inset in Fig. 1(b) shows the trajectory length distribution. Some cells divide, die, or occasionally leave the microlane during the recording period, resulting in a broad distribution of trajectory lengths. The experimental methods have been described before [36].

Single-cell velocities are estimated by position differences according to $v_{i}(t)=\left[x_{i}(t+\Delta / 2)-x_{i}(t-\Delta / 2)\right] / \Delta$ where $i=1 \ldots N$ denotes different cells. The time-dependent squared cell velocity averaged over all cells is shown in Fig. 1(b) and turns out to be rather constant for times larger than $2 \mathrm{~h}$. This indicates that cell motion is stationary for long times (nonstationary cell dynamics has been considered in previous works $[38,39])$. We therefore discard the data for the first two hours (shown in red) for all further analysis (see Appendix B for further details).

The velocity distributions for all cells are shown in Fig. 1(c), where different colors denote different cells, together with a Gaussian determined by the mean and meansquared cell velocities.

The inset demonstrates that the mean velocities $\bar{v}_{i}$ and in particular the standard deviation of the velocity for individual cells $\sigma_{i}$ scatter significantly. In Fig. 1(d) the cell velocity distributions are plotted versus the rescaled velocity coordinate $\left(v_{i}-\bar{v}_{i}\right) / \sigma_{i}$ that takes into account the individual cell statistics. The distributions are seen to follow a Gaussian (solid line) much more accurately. This is corroborated in Fig. 1(e) where we plot the cell-averaged velocity distribution versus $\left(v_{i}-\overline{\bar{v}}\right) / \bar{\sigma}$ (blue crosses) and versus $\left(v_{i}-\bar{v}_{i}\right) / \sigma_{i}$ (red spheres), where $\overline{\bar{v}}$ and $\bar{\sigma}$ are the mean velocity and the velocity standard deviation averaged over all cells. The latter way of plotting the data shows very little deviation from a Gaussian. We conclude that single cells are described within 

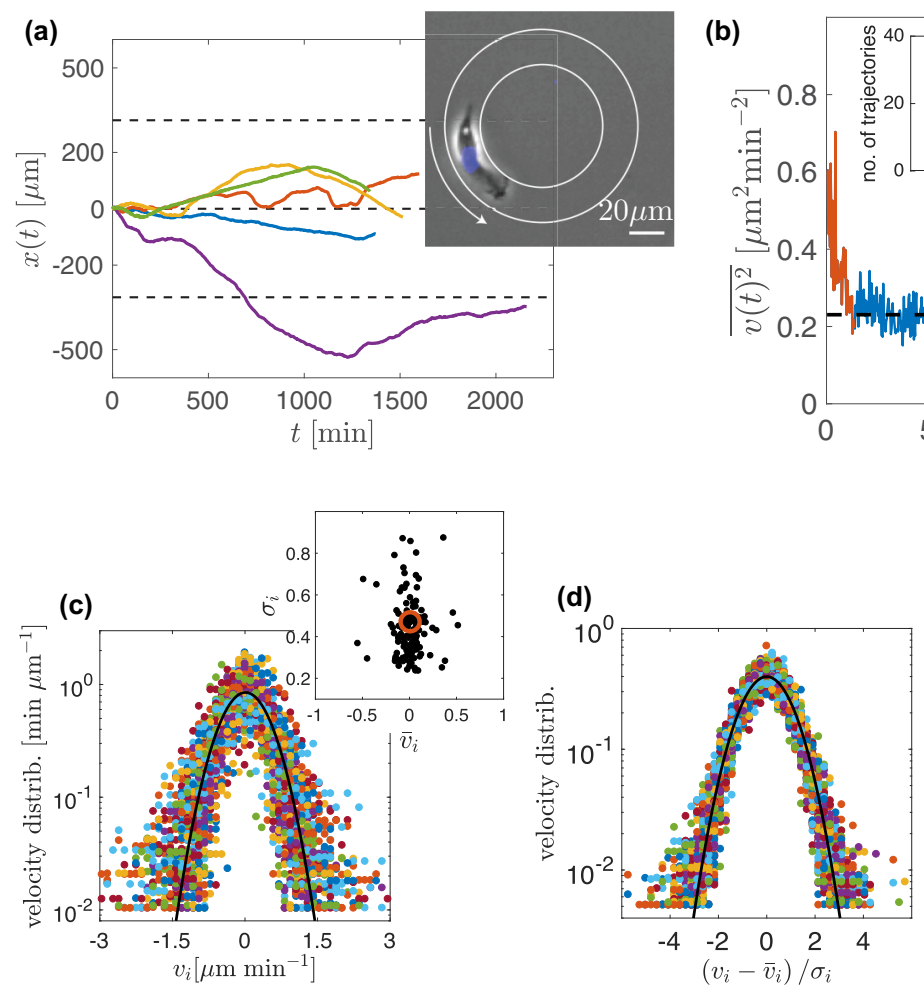

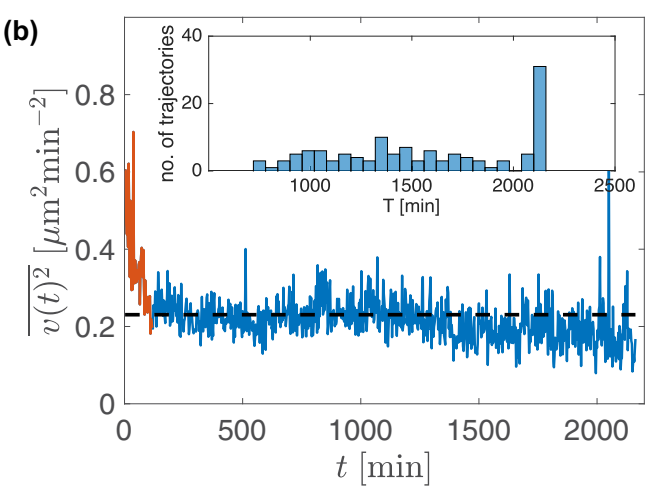

(e)

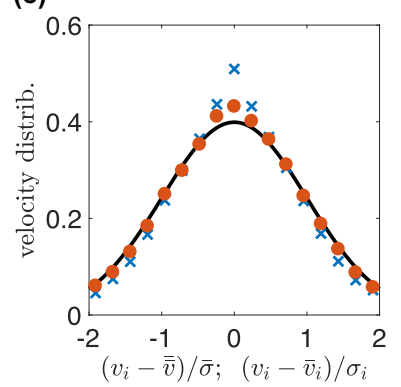

FIG. 1. Human breast cancer cell motion on circular microlanes. (a) Five randomly selected cell trajectories, where $x(t)$ is the distance coordinate along the lane. Horizontal dashed lines denote the microlane circumference of $320 \mu \mathrm{m}$. The inset depicts a phase-contrast image of a migrating cell, the fluorescently labeled nucleus shows in blue [36]. (b) Time-dependent squared velocity averaged over all cells, the horizontal black line indicates the time average. The first two hours that are colored in red are excluded from all further analysis. The inset shows the length distribution of all 125 trajectories. (c) Instantaneous velocity distribution of all cell trajectories. The black line shows a Gaussian determined by the mean and mean-squared cell velocities. The inset shows mean velocities $\bar{v}_{i}$ and velocity standard deviations $\sigma_{i}$ of single cells (black dots) as well as the average over all cells (red circle). (d) Rescaled plot of the instantaneous velocity distributions of all cell trajectories, where the velocity of each cell is shifted by its mean $\bar{v}_{i}$ and rescaled by its standard deviation $\sigma_{i}$. The black line shows a Gaussian. (e) Cell-averaged velocity distributions. Blue crosses show results plotted as a function of $\left(v_{i}-\overline{\bar{v}}\right) / \bar{\sigma}$, i.e., where the cell velocities are first averaged and then rescaled, where $\overline{\bar{v}}$ and $\bar{\sigma}$ denote the mean velocity and the velocity standard deviation averaged over all cells. When first rescaling and then averaging the data (red circles), i.e., when plotting the data as a function of $\left(v_{i}-\bar{v}_{i}\right) / \sigma_{i}$, the deviations from a Gaussian (black line) are significantly reduced.

a good approximation by Gaussian velocity distributions, that exhibit considerable variation between different cells, which, when averaged over many cells, give rise to significant nonGaussian effects, in agreement with earlier observations [17]. This means that when a theory is constructed on the single-cell level, non-Gaussian effects can for the present data set safely be neglected. This follows from the fact that multiplicative noise, i.e., when the noise term in the Langevin equation is multiplied by a velocity-dependent function, can by a nonlinear transformation be shown to produce an effective (and in general nonlinear) free energy contribution [40].

\section{B. Non-Markovian Langevin model}

We describe cell dynamics by the nonequilibrium version of the one-dimensional generalized Langevin equation (GLE) $[23,41]$

$$
\dot{v}(t)=-U[x(t)]-\int_{-\infty}^{t} d s \Gamma_{v}(t-s) v(s)+F_{R}(t),
$$

where $v(t)$ denotes the tangential velocity of the cell on the microlane and $\Gamma_{v}(t)$ is the memory kernel which accounts for non-Markovian effects and describes how the change in velocity depends on the velocity history. For Newtonian dynamics the particle mass $m$ appears in front of the acceleration term $\dot{v}(t)$, for cell dynamics the actual cell mass is irrelevant, and the prefactor of the acceleration term has been absorbed into the terms on the right side. The microtracks are homogeneous and therefore the effective potential $U[x]$ vanishes (note that memory extraction methods for general nonlinear potentials have recently been developed for the study of conformational transition kinetics [26]). The stochastic contribution $F_{R}(t)$ has zero mean $\left\langle F_{R}(t)\right\rangle=0$ and is characterized by its second moment

$$
\left\langle F_{R}(t) F_{R}(0)\right\rangle=\Gamma_{R}(t) .
$$

Since the distribution analysis of the experimental data in Fig. 1(e) suggests purely Gaussian behavior, higher-order noise correlations, which have been considered in the past $[9,12,13,19,20,38]$, are neglected. We treat the general case where the noise correlation $\Gamma_{R}(t)$ has a finite range in time, which corresponds to colored noise. For a moving cell that certainly is far from equilibrium, the two functions $\Gamma_{v}(t)$ and $\Gamma_{R}(t)$ are different in general. 

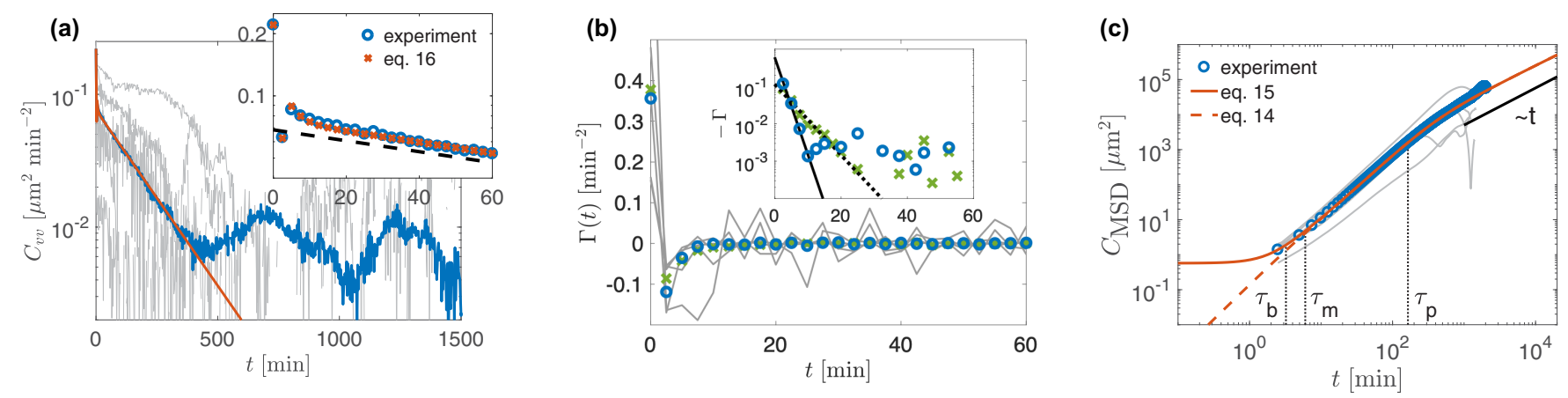

FIG. 2. (a) Cell-averaged velocity autocorrelation function $C_{v v}(t)$ (blue line) compared with the model prediction Eq. (16) (red line). Thin gray lines denote data for the five individual cells shown in Fig. 1(a). The inset shows short-time results on the full experimental time resolution of $\Delta=2.5 \mathrm{~min}$, the broken black line denotes the PRW model prediction which is a straight line in the log-linear plot. (b) Memory kernel $\Gamma(t)$ (blue circles) directly extracted from the experimental cell-averaged $C_{v v}(t)$ data in (a). Thin gray lines denote results for the five individual cells shown in (a). The average of the kernels extracted from all single-cell trajectories is denoted by green crosses. The inset shows $-\Gamma(t)$ at short time on a log-linear scale together with single exponential fits to the first four (eight) data points of the negative tails of the kernels, shown by solid (dashed) black lines. (c) Mean-squared cell displacements averaged over all cells (blue data points) together with the analytical model result with and without localization noise according to Eqs. (15) and (14), respectively. The vertical lines mark the ballistic time $\tau_{b}$, the memory time $\tau_{m}$, and the persistent time $\tau_{p}$. Thin gray lines denote experimental data for the five individual cells shown in (a) and (b).

The linear Langevin equation is characterized by the VACF

$$
C_{v v}(t)=\langle v(0) v(t)\rangle,
$$

from which positional correlation functions, the meansquared displacement, as well as all higher moments can be calculated. The Fourier-transformed VACF resulting from the Langevin equation (1) reads as

$$
\tilde{C}_{v v}(\omega)=\frac{\tilde{\Gamma}_{R}(\omega)}{\left[i \omega+\tilde{\Gamma}_{v+}(\omega)\right]\left[-i \omega+\tilde{\Gamma}_{v+}(-\omega)\right]},
$$

where Fourier transforms are given by $\tilde{f}(\omega)=$ $\int_{-\infty}^{\infty} d t e^{-i \omega t} f(t)$ and $\tilde{f}_{+}(\omega)=\int_{0}^{\infty} d t e^{-i \omega t} f(t)$ denotes the single-sided Fourier transform (the derivation is given in Appendix C).

Our general non-Markovian model reduces to the PRW model in the Markovian (i.e., white noise) limit, i.e., when the Fourier-transformed memory function $\tilde{\Gamma}_{v+}(\omega)$ and the noise correlator $\tilde{\Gamma}_{R}(\omega)$ are constant; in this case the VACF $C_{v v}(t)$ decays as a single exponential. The experimental cellaveraged VACF in Fig. 2(a) (blue line) decays exponentially over $500 \mathrm{~min}$ and is dominated by noise for longer times, the inset demonstrates deviations from single-exponential decay over the initial $20 \mathrm{~min}$ by comparison with the straight broken black line in the log-linear plot. This shows that the cells do not behave according to the PRW model but that either finite memory or colored noise is present in the data [we will later show that deviations from PRW behavior are also present on the single-cell level and therefore the deviations seen in the cell-averaged VACF in Fig. 2(a) are not caused by variations among different cells]. As we will discuss in more detail later on, the discontinuous behavior of the VACF for the first two data points in the inset in Fig. 2(a) is explained by noise stemming from the localization of the cell nucleus [17].

At this point, a fundamental problem is encountered: While the Fourier-transformed VACF $\tilde{C}_{v v}(\omega)$ in Eq. (4) is uniquely determined by the memory function $\tilde{\Gamma}_{v+}(\omega)$ and noise correlation $\tilde{\Gamma}_{R}(\omega)$, we cannot uniquely extract these two functions from $\tilde{C}_{v v}(\omega)$. For a general Gaussian process, the velocity Green's function, from which all correlation functions, conditional as well as unconditional averages can be derived, is uniquely defined by the VACF (see Appendix D). This in turn implies that the Fourier-transformed VACF $\tilde{C}_{v v}(\omega)$ contains complete information on the cell migratory characteristics. Thus, based on one-dimensional cell trajectories, the problem is underdetermined. To proceed with our analysis, we for the time being restrict the parameter space and collapse the two functions $\Gamma_{v}(t)$ and $\Gamma_{R}(t)$ into one (we will discuss how to undo this collapse later on). In analogy with the equilibrium scenario, we define a substitute memory function by

$$
\Gamma(t) \equiv \Gamma_{v}(t)=\Gamma_{R}(t) / B,
$$

where $B$ will be later shown to correspond to the meansquared cell velocity. From Eq. (4) it follows that the VACF predicted by the full model [i.e., when $\Gamma_{v}(t)$ and $\Gamma_{R}(t)$ are independent of each other] and by the substitute model [when Eq. (5) holds] are the same, if the condition

$$
\frac{B \tilde{\Gamma}(\omega)}{\left|i \omega+\tilde{\Gamma}_{+}(\omega)\right|^{2}}=\frac{\tilde{\Gamma}_{R}(\omega)}{\left[i \omega+\tilde{\Gamma}_{v+}(\omega)\right]\left[-i \omega+\tilde{\Gamma}_{v+}(-\omega)\right]}
$$

is satisfied, where the relation $\tilde{\Gamma}(\omega)=2 \operatorname{Re} \tilde{\Gamma}_{+}(\omega)$ holds. $\tilde{\Gamma}_{R}(\omega)$ follows from Eq. (6) as a unique function of $\tilde{\Gamma}_{v+}(\omega)$ and $\tilde{\Gamma}_{+}(\omega)$, so we conclude that there are infinitely many combinations of $\tilde{\Gamma}_{v+}(\omega)$ and $\tilde{\Gamma}_{R}(\omega)$ that for given $\tilde{\Gamma}_{+}(\omega)$ satisfy Eq. (6).

In fact, the substitute kernel $\Gamma(t)$ can be uniquely determined from the experimental VACF. To see this, we note that the GLE equation (1) can be rewritten as

$$
C_{v v}(t)-C_{v v}(0)=-\int_{0}^{t} d s G(t-s) C_{v v}(s),
$$

where $G(t) \equiv \int_{0}^{t} \Gamma\left(t^{\prime}\right) d t^{\prime}$ is the running integral over the substitute memory function. Given an experimental VACF $C_{v v}(t)$ as input, Eq. (7) can be inverted which thereby uniquely determines $G(t)[41,42]$, from which the memory function $\Gamma(t)$ 
follows by differentiation. Numerical details are explained in Appendix E.

The memory kernel $\Gamma(t)$ extracted from the cell-averaged VACF is shown in Fig. 2(b) (blue open circles), while the green crosses denote the average over memory kernels extracted from VACFs of individual cells. The agreement between the two different kernel functions is rather good, the inset demonstrates that the kernels decay with similar characteristic timescales of 1.7 and $4.6 \mathrm{~min}$, respectively, obtained from fitting single-exponential functions to the tails. Thus, we conclude that the functional form of the kernel is not influenced significantly by cell-to-cell variations of the VACF and thus constitutes a robust feature of the cell dynamics. The thin gray lines denote memory kernels for the five individual cell trajectories shown in Fig. 1(a). On the experimental time resolution, the kernel consists of a positive contribution at time zero and a negative tail, which, as shown in the inset, is a single exponential. The data thus suggest a generic kernel

$$
\Gamma(t)=2 a \delta(t)+b e^{-t / \tau_{m}}
$$

with $b<0$. The standard PRW model is recovered in the limit $b=0$. The simple yet faithful model suggested by the data thus contains a total of four parameters: $a, b$, and $\tau_{m}$ as defined in Eq. (8), and $B$, as defined in Eq. (5). The VACF for this model follows as

$$
C_{v v}(t)=\frac{B \tau_{p}\left(\tau_{m}-\tau_{b}\right)}{\tau_{m}\left(\tau_{p}-\tau_{b}\right)} e^{-t / \tau_{b}}+\frac{B \tau_{b}\left(\tau_{p}-\tau_{m}\right)}{\tau_{m}\left(\tau_{p}-\tau_{b}\right)} e^{-t / \tau_{p}},
$$

where the two relaxation times are defined by

$$
\tau_{p, b}=\frac{1+\tau_{m} a \pm \sqrt{\left(1+\tau_{m} a\right)^{2}-4 b \tau_{m}^{2}-4 a \tau_{m}}}{2\left(a+\tau_{m} b\right)}
$$

with $\tau_{p}$ the persistence time and $\tau_{b}<\tau_{p}$ the ballistic time. The mean-squared velocity is given by

$$
C_{v v}(0)=B,
$$

which directly follows from Eqs. (4) and (5) (see Appendix C). The cell diffusion constant $D$ is is given by an integral over the VACF, which leads to

$$
D=\frac{\tilde{C}_{v v}(0)}{2}=\frac{B}{a+b \tau_{m}} .
$$

The two relations (11) and (12) effectively reduce the number of parameters from four to two, if experimental estimates for $C_{v v}(0)$ and $D$ are used.

\section{Discretization effects and localization noise}

Since the extracted memory time $\tau_{m}$ is of the order of the discretization time $\Delta=2.5 \mathrm{~min}$, discretization effects, which invariably come in when positional data at finite-time differences are used to calculate velocities and VACFs, are expected to perturb the memory extraction. A second effect we have not discussed so far comes from the localization noise which is produced when the fluorescence intensity profile from the labeled nucleus is projected onto a single positional variable. In order to account for discretization and localization noise effects, which are difficult to include in the memory extraction, we complement our data-based memory extraction method by forward modeling of the experimental VACF. For this we first define the mean-squared displacement

$$
C_{\mathrm{MSD}}(t)=\left\langle[x(t)-x(0)]^{2}\right\rangle,
$$

which follows from the VACF by a double integration according to $C_{\mathrm{MSD}}(t)=2 \int_{0}^{t} d t^{\prime} \int_{0}^{t^{\prime}} d t^{\prime \prime} C_{v v}\left(t^{\prime \prime}\right)$. From $C_{v v}(t)$ in Eq. (9) we obtain [28]

$$
\begin{aligned}
C_{\mathrm{MSD}}(t) /(2 D)= & t+\frac{\tau_{b}\left(\tau_{m}-\tau_{b}\right)}{\tau_{p}-\tau_{b}}\left(e^{-t / \tau_{b}}-1\right) \\
& +\frac{\tau_{p}\left(\tau_{p}-\tau_{m}\right)}{\tau_{p}-\tau_{b}}\left(e^{-t / \tau_{p}}-1\right) .
\end{aligned}
$$

The localization noise of the cell nucleus position can be modeled by adding a random variable $x_{\text {loc }}$ to the position so that the model prediction for the experimentally measured position reads as $x_{\text {expt }}(i \Delta)=x(i \Delta)+x_{\text {loc }}(i \Delta)$. Assuming the localization noise to be uncorrelated over time and characterized by the deviation $\sigma_{\text {loc }}$, we obtain for the MSD $[17,43]$

$$
C_{\mathrm{MSD}}^{\mathrm{expt}}(i \Delta)=C_{\mathrm{MSD}}(i \Delta)+2\left(1-\delta_{i 0}\right) \sigma_{\mathrm{loc}}^{2} .
$$

The VACF follows by the discrete second derivate according to

$$
C_{v v}^{\operatorname{expt}}(i \Delta)=\frac{C_{\mathrm{MSD}}^{\mathrm{expt}}[(i+1) \Delta]-2 C_{\mathrm{MSD}}^{\mathrm{expt}}(i \Delta)+C_{\mathrm{MSD}}^{\mathrm{expt}}[(i-1) \Delta]}{2 \Delta^{2}}
$$

and includes both discretization and localization noise effects (see Appendix F). With the experimental values for the meansquared velocity $C_{v v}^{\text {expt }}(0)=0.22 \mu \mathrm{m}^{2} / \mathrm{min}^{2}$ and the diffusion constant $D^{\text {expt }}=12.3 \mu \mathrm{m}^{2} / \mathrm{min}$, calculated via integrating the discrete experimental VACF in Fig. 2(a), there are, including the localization noise strength $\sigma_{\text {loc }}$, three parameters. These are determined via a fit of Eq. (16) to the experimental VACF in Fig. 2(a). The fit is robust since the remaining fit parameters are determined by distinct features of the VACF, namely, the nonmonotonicity in the first three data points due to the localization noise and the two decay times of the VACF set by $\tau_{b}$ and $\tau_{p}$ (see Appendix $G$ for details of the fit and the evaluation of $D^{\text {expt }}$ ). The best fit is shown in Fig. 2(a) by a red line and in the inset by red crosses, it describes the experimental VACF perfectly on all timescales. We note that the fit values for the memory parameters $a, b$, and $\tau_{m}$ differ from the values directly extracted from the experimental data and shown in Fig. 2(b), which clearly indicates the importance of discretization and localization noise effects.

In Fig. 2(c) the experimental MSD data (blue circles) are compared with the prediction from the fit to the cell-averaged VACF including localization effects according to Eq. (15) (solid red line), exhibiting perfect agreement. We also show theory results for the case where we leave all parameters fixed but set $\sigma_{\text {loc }}=0$ (red broken line). By comparison of the two predictions we see that localization noise gives rise to a saturation of the MSD for short times. Clearly, the deviation between these two scenarios is only discernible in the first experimental MSD data point. In contrast, for the VACF data in Fig. 2(a), localization noise has a more drastic effect and leads to a characteristic nonmonotonicity in the first three data 
(a)

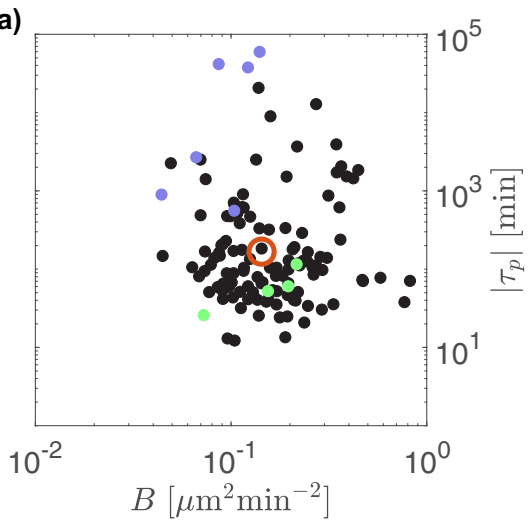

(d)

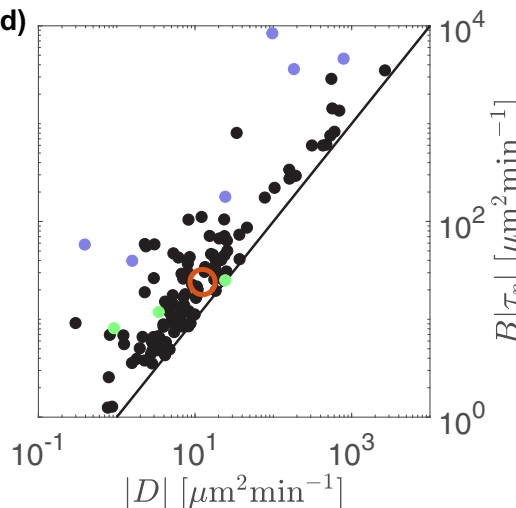

(b)

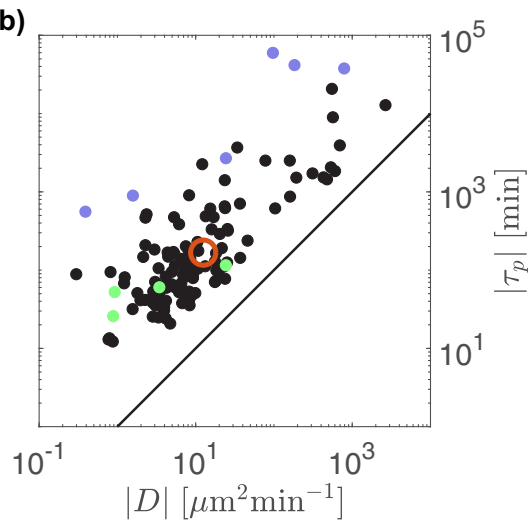

(e)

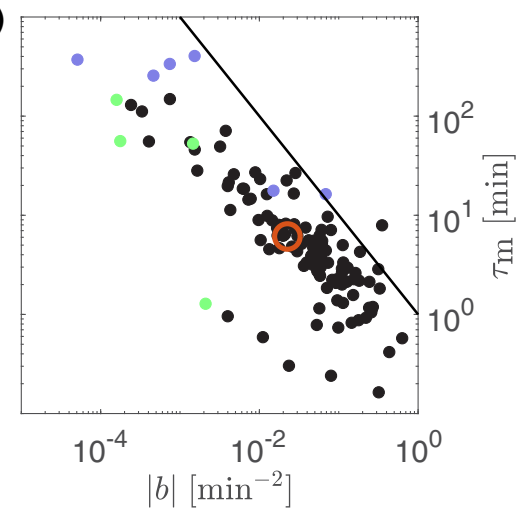

(c)

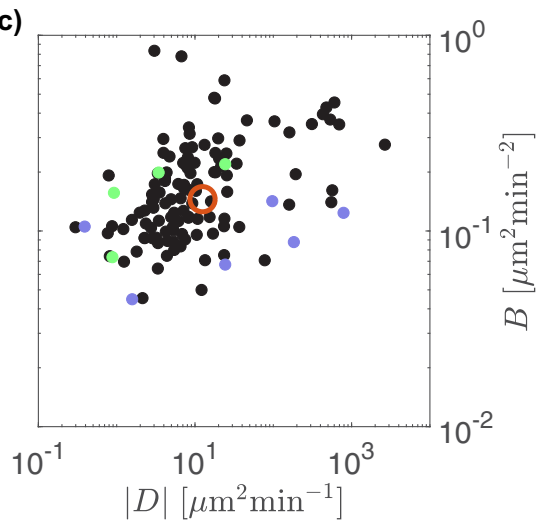

(f)

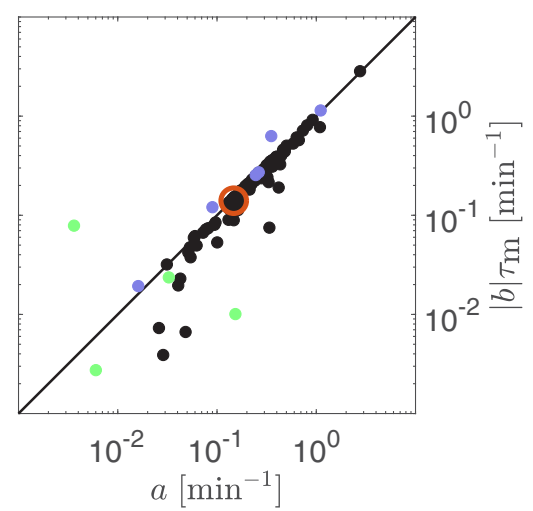

FIG. 3. Correlation analysis of single-cell parameters obtained from fits of Eq. (16) to single-cell VACFs. Cell data for which $b$ is positive are shown as green circles, blue circles denote cells for which $D$ according to Eq. (12) is negative, black circles denote the majority of cells for which $b$ is negative and $D$ positive. Red open circles denote the result from the analysis of the cell-averaged VACF. (a)-(c) Correlations between the diffusivity $D$, squared cell velocity $B$, and persistence time $\tau_{p}$. Comparison with the solid line in (b) indicates weak linear scaling. (d) Scaling plot of $B \tau_{p}$ versus $D$, the PRW prediction $D=B \tau_{p}$ is denoted by a solid line and constitutes an upper bound to the single-cell diffusivity. (e) The plot of the memory time $\tau_{m}$ versus the amplitude of the exponential memory contribution $|b|$ reveals a roughly inverse linear scaling (denoted by a solid line), which suggests that the integrated contribution of the exponential memory term to the friction, $|b| \tau_{m}$, is rather constant. (f) Scaling plot of $|b| \tau_{m}$ versus $a$, demonstrating that the two contributions to the memory function defined in Eq. (8) have equal integrated weight.

points. Localization noise thus has a more significant effect on VACF data compared to MSD data.

The timescales $\tau_{b}=3 \mathrm{~min}, \tau_{m}=6 \mathrm{~min}$, and $\tau_{p}=160 \mathrm{~min}$ are indicated by vertical colored lines.

\section{Correlation analysis of single-cell parameters}

One goal of single-cell motility analysis is the comparison and classification of individual cells. In Figs. 3(a)-3(c) we present the correlations between the single-cell results for the diffusivity $D$, the squared cell velocity $B$, and the persistence time $\tau_{p}$, as they follow from our fits to the single-cell VACF data using the GLE defined by Eqs. (1), (5), and (8). These three observables can be straightforwardly extracted from experimental VACF by direct fitting to the PRW model and similar correlation plots have been presented in previous studies $[17,44]$. The advantage of our indirect extraction via fit to the GLE is that memory, discretization and localization noise effects, which strongly perturb $B$, are properly accounted for.

A very weak positive correlation between $\tau_{p}$ and $B$ is seen in Fig. 3(a), meaning that cells with a larger instantaneous velocity also show a larger persistence time, in agreement with previous results [44] (where, however, the persistence time was defined differently). There is a pronounced linear correlation between $\tau_{p}$ and $D$ over three orders of magnitude in Fig. 3(b), which was also noted in literature before [17], while there is again only little correlation between $B$ and $D$ in in Fig. 3(c). The main difference between these observables is that $B$ varies little from cell to cell, spanning only a decade, while $D$ and $\tau_{p}$ vary over more than three orders of magnitude. The PRW model predicts the diffusivity $D$ to be given by $D=B \tau_{p}$ [7], which in Fig. 3(d) is shown to be obeyed by our data very nicely over the entire range of individual cell data. We conclude that the quasilinear correlation between $\tau_{p}$ and $D$ in Fig. 3(b) is a consequence of the PRW scaling $D=B \tau_{p}$ in Fig. 3(d), produced by the fact that $B$ shows little cell-to-cell variation, and does not necessarily present a distinct property of cell migratory behavior.

Deviations of our single-cell cell data from the PRW model in the scaling plot Fig. 3(d) are rather mild. This means that the PRW model is pretty accurate for the long-time cell migration as described by diffusivity $D$, persistence time $\tau_{p}$, and squared cell speed $B$. It is only the short-time behavior of the VACF, shown in the inset of Fig. 2(a), that reveals 
the deviations from the PRW model. In Fig. 3(d) we observe that the single-cell diffusivity is systematically smaller than predicted by the PRW model, which is due to the characteristic form of the cell memory function, as will be explained below.

In Fig. 3(e) we show a correlation plot of the memory time $\tau_{m}$ versus the amplitude $|b|$ of the exponential term in the memory function defined in Eq. (8). We observe a large spread of the memory time over three orders of magnitude, from fractions of a minute to few hours, and at the same time a pronounced anticorrelation between $\tau_{m}$ and $|b|$ : cells that are characterized by long memory times $\tau_{m}$ show small amplitudes $|b|$. This anticorrelation suggests the correlation plot of the product $|b| \tau_{m}$ versus the amplitude $a$ of the delta function in the memory function defined in Eq. (8), shown in Fig. 3(f). The almost perfect linear scaling $a=|b| \tau_{m}$, denoted by the straight line, mathematically implies that the memory function of cells is tuned such that the two relaxation times $\tau_{b}$ and $\tau_{p}$, defined in Eq. (10), are maximally different from each other. The other parameters of our non-Markovian cell model, $a, b, B, \tau_{m}$, exhibit rather weak correlations, as shown in Appendix $\mathrm{H}$.

In conclusion, single-cell memory parameters allow to compare single cells in an unbiased fashion. The spread of the parameters in Fig. 3 is rather large; it is at the present stage not clear whether the pronounced scattering stems from cell heterogeneity or from the shortness of the trajectories. However, it is clearly seen that the data do not fall into distinct clusters. The single-cell parameters are centered around the parameters fitted to the cell-averaged VACF (red circles). This corroborates that the memory function model we extract is not modified by the cell-to-cell variation in a significant fashion, in line with the good comparison of the memory function extracted from the cell-averaged VACF and the average over the memory functions extracted from single-cell data in Fig. 2(b).

\section{E. Timescale analysis of MSD}

The model with a negative exponential kernel contribution, Eq. (8), can be analyzed in closed form and exhibits a characteristic persistence enhancement. In fact, in the limit $\tau_{b}<\tau_{m}<\tau_{p}$ the model exhibits four different regimes [45]:

$$
C_{\mathrm{MSD}}(t) \sim \begin{cases}t^{2}, & t \ll \tau_{b} \\ t, & \tau_{b} \ll t \ll \tau_{m} \\ t^{2}, & \tau_{m} \ll t \ll \tau_{p} \\ t, & \tau_{p} \ll t\end{cases}
$$

So there is a short-time regime $t \ll \tau_{b}$ where motion is ballistic, an intermediate regime for $\tau_{b} \ll t \ll \tau_{m}$ where motion is diffusive, a second intermediate regime for $\tau_{m} \ll t \ll \tau_{p}$ where memory effects induce persistent motion, and finally the asymptotic diffusive regime for $\tau_{p} \ll t$. This multiscale migration behavior is illustrated in Fig. 4, which shows the transient MSD exponent

$$
\alpha(t)=\frac{d \ln C_{\mathrm{MSD}}(t)}{d \ln t} .
$$

For $B, a$, and $b$ we use the values resulting from the fit to the cell-averaged VACF in Fig. 2(a) and present $\alpha$ as a function of time $t$ and a variable memory time $\tau_{m}$. The best-fit value $\tau_{m}=6 \mathrm{~min}$ is indicated by a horizontal black broken line.

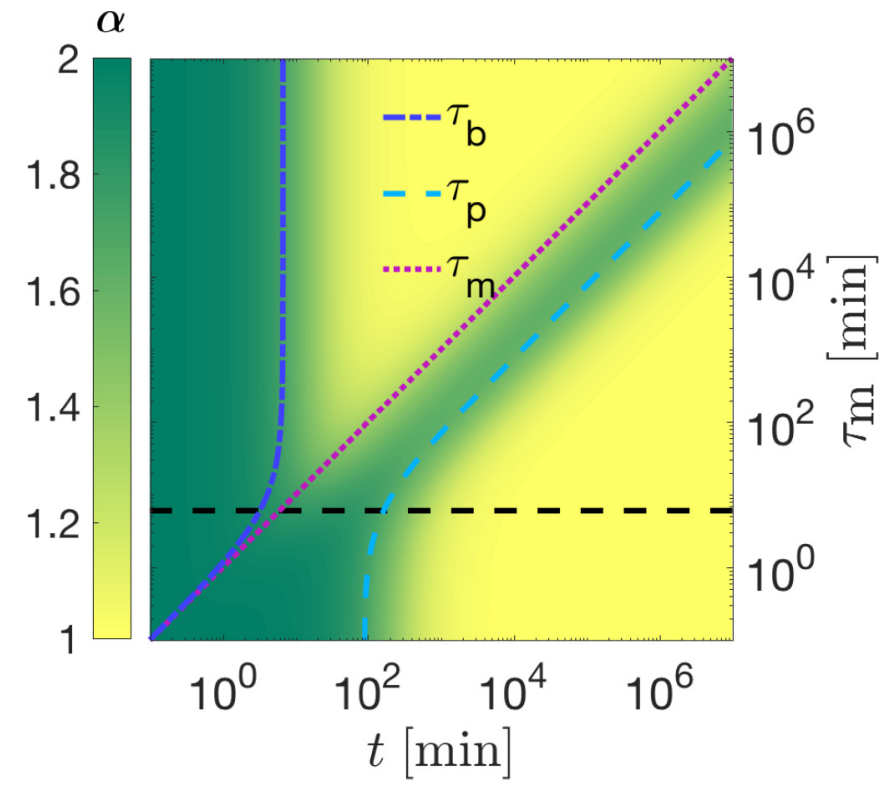

FIG. 4. Phase diagram for the MSD exponent $\alpha$ (denoted by the green-yellow color scale) as a function of time $t$ and the memory time $\tau_{m}$. The memory time from the fit to the cell-averaged VACF in Fig. 2(a) is shown as a horizontal dashed line. For long memory times $\tau_{m}$, an intermediate diffusive regime ( $\alpha \approx 1$, shown in yellow) appears between the short-time ballistic (left green region) and the long-time persistent regimes (right green region) where $\alpha \approx 2$.

As $\tau_{m}$ increases, an intermediate diffusive regime (yellow region) at intermediate times develops and a second persistent regime (green strip to the right) at long times appears. This persistent regime appears at times that are substantially longer than the memory time, so we conclude that memory induces persistent motion at longer times than the memory time itself. For the actual memory time extracted from the averaged cell data, $\tau_{m}=6 \mathrm{~min}$, the short-time ballistic and the long-time persistent regimes merge, but from the scaling diagram it transpires that a small increase of the memory time induces an intermediate diffusive regime and extended persistent motion over much longer timescales. The cancer cells we study are therefore close to a transition to the full four-scale dynamic behavior as described by Eq. (17). The presence of the intermediate diffusive regime in the time range for $\tau_{b} \ll t \ll \tau_{m}$ also explains why for the cell data in Fig. 3(d) the PRW model constitutes an upper bound for the cell diffusivity: this is so because the intermediate diffusive behavior in Eq. (17) lowers the MSD compared to a purely ballistic motion.

\section{F. Reconstruction of equivalent Markovian models}

Our cell motility analysis utilizes the GLE with a single substitute memory function $\Gamma(t)$, defined in Eq. (5), which by extraction from experimental data is shown to exhibit an exponentially decaying negative contribution according to Eq. (8). There are infinitely many different non-Markovian nonequilibrium models, characterized by two different functions $\Gamma_{v}(t)$ and $\Gamma_{R}(t)$, that according to Eq. (6) produce the same VACF.

Non-Markovian effects arise from Markovian manybody systems by integrating out degrees of freedom [23], 
conversely, for any non-Markovian GLE a Markovian higherdimensional substitute model can be constructed. In Appendix I we demonstrate that the GLE with a single substitute memory function $\Gamma(t)$ given by Eq. (8) corresponds to an equilibrium Markovian system of two degrees of freedom with nondiagonal friction coupling. This of course does not imply that cell migration is an equilibrium process, dissipative effects would in such a model be expected to show up on a hidden more microscopic level. As explained in Appendix J, it is also possible to construct different equivalent two-dimensional nonequilibrium Markovian models. One particularly simple example of a nonequilibrium model that reproduces the experimental VACF and at the same time allows to be written in terms of a coupled, stable system of Markovian rate equations, is given by

$$
\Gamma_{v}(t)=2 a_{v} \delta(t), \quad \Gamma_{R}(t)=2 a_{R}^{2} \delta(t)+\frac{b_{R}^{2}}{\tau_{R}} e^{-t / \tau_{R}},
$$

which corresponds to a Langevin equation with an instantaneous friction function $\Gamma_{v}(t)$ and a colored-noise correlator $\Gamma_{R}(t)$. The coefficients $a_{v}, a_{R}, b_{R}, \tau_{R}$ follow from Eq. (6) as $\tau_{R}=\tau_{p}, a_{v}=1 / \tau_{b}, a_{R}^{2}=B a, b_{R}^{2}=B\left(\tau_{p} / \tau_{b}-\tau_{m} a\right) / \tau_{m}$ (see Appendix $\mathrm{J}$ for details). Interestingly, the persistence time $\tau_{p}$, which is the longest timescale in the system, equals the correlation time $\tau_{R}$ of the active noise in Eq. (19). The nonequilibrium non-Markovian Langevin defined by Eq. (19) can be derived from the two coupled Markovian equations of motion

$$
\begin{aligned}
& \dot{\eta}(t)=-\tau_{R}^{-1} \eta(t)+\tau_{R}^{-1} \xi_{1}(t), \\
& \dot{v}(t)=-a_{v} v(t)+a_{R} \xi_{0}(t)+b_{R} \eta(t),
\end{aligned}
$$

where $\eta(t)$ is an additional fluctuating degree of freedom. The two white Gaussian noise variables $\xi_{i}(t)$ with $i=0,1$ are defined by correlators $\left\langle\xi_{i}(t) \xi_{j}\left(t^{\prime}\right)\right\rangle=2 \delta_{i j} \delta\left(t-t^{\prime}\right)$. The effective random force entering the GLE equation (1) for the single variable $v(t)$ is given by $F_{R}(t)=a_{R} \xi_{0}(t)+b_{R} \eta(t)$ and reproduces the random correlation $\Gamma_{R}(t)$ given in Eq. (19), as is shown in Appendix J. This model has been studied amply in literature and corresponds to the underdamped version of the active Ornstein-Uhlenbeck process [46]. In fact, a collection of particles described by the correlators in Eq. (19) undergoes a motility-induced phase separation for a suitable choice of parameter values $[47,48]$. We have thus demonstrated that the motion of the cancer cells studied by us can be mapped onto a nonequilibrium particle model that is well known in literature. We hasten to add that this mapping is not unique and that there are other equilibrium and nonequilibrium Markovian models on which we can map our extracted substitute kernel $\Gamma(t)$, in Appendices $\mathrm{I}$ and $\mathrm{J}$ we show alternative equilibrium and nonequilibrium Markovian models that have similar complexity as the model defined in Eq. (19).

\section{CONCLUSIONS}

The goal of single-cell motility modeling is to extract parameters from single-cell trajectories that allow to characterize, compare, classify, and, to a certain degree, understand cell migration. In traditional modeling approaches, this goal is achieved by defining a migratory model and by extracting parameters of that model by fitting to experimental data.
While such approaches have produced remarkable insights into the dynamics of cells $[17,44]$, the large number of different random walk models renders the extracted parameters not unique. This is enhanced by the fact that single-cell data show large cell-to-cell variation and pronounced single-cell noise due to the typically short trajectory length.

We here introduce an alternative modeling approach, which is based on the generalized Langevin equation that includes an arbitrary memory friction function $\Gamma_{v}(t)$ and an arbitrary colored-noise correlator $\Gamma_{R}(t)$. By construction, our cell motion model contains many previously introduced random walk models; as the main advantage of our approach, we extract the memory function in a data-driven approach from the trajectory time series. We do not impose a certain model on the cell data, but we rather let the cell migration data reveal to us the model that best describes the trajectories.

In the first part we extract a substitute memory function $\Gamma(t)$ directly from the cell VACF. The memory function contains an exponentially decaying negative friction contribution, which demonstrates that the cell dynamics shows small but significant deviations from the standard PRW model, according to which the memory function would simply be a delta function. The presence of a negative exponentially decaying friction contribution is interesting because the migratory dynamics produced by such a model is equivalent to the underdamped version of the active Ornstein-Uhlenbeck process, as we show in Appendix J. This is remarkable since the fact that cancer cell motion is equivalent to this active particle model is data driven and follows directly from the cell trajectories.

Based on the trajectories alone, it is not possible to uniquely extract the memory friction function $\Gamma_{v}(t)$ and the noise correlator $\Gamma_{R}(t)$; this is not a shortcoming of our model or the methods we use, but rather follows from the limited amount of information contained in unbiased one-dimensional trajectories. In future studies, it would be interesting to subject cells to external perturbations (such as chemical gradients or confining forces), which would allow to determine response functions and thereby to extract $\Gamma_{v}(t)$ and $\Gamma_{R}(t)$ separately. This would help to characterize the nonequilibrium character of biological systems in more detail. In such studies, presumably non-Gaussian velocity distributions will be encountered, which can be dealt with by the nonlinear kernel extraction methods that we developed earlier [25,26]. Alternatively, multidimensional cell marker data directly signal detailed balance violation and thereby allow to detect the nonequilibrium character of the system dynamics [49]. In fact, multidimensional data can be analyzed by straightforward adaption of the methods presented in this paper.

Clearly, it would be desirable to relate the characteristics of the memory function we extract from cell trajectories to biochemical processes in the cell. Numerous experimental and theoretical studies considered cell protrusion and cell polarization dynamics and in particular investigated the connection to the underlying dynamics of the extracellular matrix, the actin network, the cellular polarity machinery, and integrin trafficking [50-52]. Interestingly, we find the negative friction component of the memory kernel to decay over a few minutes, which is similar to the timescale observed for the cell protrusion kinetics [51], which could indicate a possible connection. However, we note that the 
memory time distribution of individual cells in Fig. 3(e) is extremely broad and shows memory times $\tau_{m}$ from less than a minute to hours, which does not seem to be mirrored by an equally broad distribution of cell protrusion timescales. Indeed, the connection between timescales of the dynamics of cell components (such as the actin network or cell protrusions) and the memory timescale that characterizes the overall cell motion is presumably rather complex: This is demonstrated by the simple Markovian models derived in Appendix J, where the memory timescale $\tau_{m}$ depends not only on the separate timescales of the coupled coordinates, but also on the coupling strength between the coordinates in a complex manner. In fact, the connection of the memory function timescales to underlying cell component dynamics could be addressed experimentally by studying the effect of chemical agents that interfere with, e.g., the cytoskeleton on the memory function or, even more directly, by using multiple cell markers that couple to different parts of the cell migratory machinery. In this context, experimental data with finer time discretization, for which alternative staining methods need to be employed, would allow to better resolve the short-time behavior of the extracted memory kernel functions.

Future experiments with different cell lines will demonstrate the potential of our memory kernel extraction technique for cell comparison and classification. Our methods can be applied to all different kinds of living and synthetic active objects, comparison of the extracted memory functions will reveal whether the negative friction component of the memory function found in this study is a general hallmark of active systems.

\section{ACKNOWLEDGMENTS}

We gratefully acknowledge financial support from the Deutsche Forschungsgemeinschaft (DFG) via Grant No. SFB 1114 project B3. This project received funding from the European Union's Horizon 2020 Research and Innovation Programme under Grant Agreement No. 674979-NANOTRANS and from the European Research Council (ERC) under the European Union's Horizon 2020 research and innovation program under Grant Agreement No. 835117.

\section{APPENDIX A: EXPERIMENTS}

\section{Microcontact printing}

Polydimethylsiloxane (PDMS) stamps were treated with UV light (PSD-UV, Novascan) for 5 min and incubated for $45 \mathrm{~min}$ in a $50-\mu \mathrm{g} / \mathrm{ml}$ fibronectin solution (Yo proteins). Next, stamps were washed with deionized water, dried, and placed on a plastic dish ( $\mu$-Dish, Ibidi), which had been treated with UV light for $15 \mathrm{~min}$. A droplet of a $1 \mathrm{mg} / \mathrm{ml}$ poly-Llysine-grafted polyethylene glycol (PLL-PEG) (SuSoS) solution (dissolved in $10 \mathrm{mM}$ HEPES containing $150 \mathrm{mM} \mathrm{NaCl}$ ) was placed at the edge of the stamps and drawn into the spaces between surface and stamp by capillary action. Stamps were removed and a glass coverslip was placed on the dish surface to ensure complete coverage of the surface with PEG solution. After a 30-min incubation, the coverslip was removed and the surface was washed three times with PBS and stored in PBS until cells were seeded.

\section{Cell cultures}

MDA-MB-436 breast cancer cells were cultured in DMEM-F12 medium (c.c.pro) containing 10\% fetal bovine serum (Invitrogen) and $2.5 \mathrm{mM} \mathrm{L}$-glutamine (c.c.pro) at $37^{\circ} \mathrm{C}$ in a $5 \% \mathrm{CO}_{2}$ atmosphere. For experiments about 10000 cells were seeded per dish and the medium was exchanged after $2 \mathrm{~h}$ to L15 medium without phenol red with $25 \mathrm{nM}$ Hoechst added.

\section{Time-lapse microscopy}

Cells were monitored using an inverted microscope equipped with an $10 \times$ objective (Nikon TI), an automated stage (Märzhäuser), a sCMOS camera (PCO), and a heating chamber to keep cells at $37^{\circ} \mathrm{C}$ (Okolab). Every $2.5 \mathrm{~min}$, phase-contrast and fluorescence images of the nuclei were acquired.

\section{Cell tracking}

Single cells on the microlanes where manually identified. A band pass filter was applied to the fluorescence images of the nucleus. Then, a binary image was generated using a threshold. The geometric center of the nucleus was used as the cell position. Cell tracking was terminated in the case of cell division, cell death, or when cells spanned over the middle part of the ring or migrated out of the microlane. The center of the ring-shaped microlane was determined by fitting a circle to the cell positions to allow a transformation to polar coordinates.

\section{APPENDIX B: STATIONARITY}

To check whether the data are consistent with the assumption of stationarity, we estimate the second moment of the velocities by averaging over all cells

$$
\overline{v^{2}(t)}=\frac{1}{n(t)} \sum_{i} \Theta\left(T_{i}-t\right) v_{i}^{2}(t),
$$

where $n(t)$ denotes the number of cell trajectories which are at least of length $t$ and $T_{i}$ denotes the total length of trajectory $i$. In the above expression, the individual trajectories all start at time $t=0$. Since not all trajectories are of the same length, the average is taken only over a fraction of the total number of cells for large $t$ in the above estimate. To investigate the behavior near the end of all trajectories, we also estimate the second moment with the trajectories shifted such that they all end at $t=0$ :

$$
\overline{v^{2}(t-T)}=\frac{1}{n(T-t)} \sum_{i} \Theta\left(T_{i}-T+t\right) v_{i}^{2}(T-t) .
$$

The estimate $\overline{v^{2}(t)}$ shows a peak during the first two hours and is rather constant after that (see Fig. 5). This peak is smeared out in the shifted estimate $\overline{v^{2}(t-T)}$, which otherwise is rather constant and does not show any anomaly toward the trajectory end. Nonstationary behavior is thus only discernible in the first two hours of the trajectories, which are therefore discarded for all further analysis, as noted in the main text. 

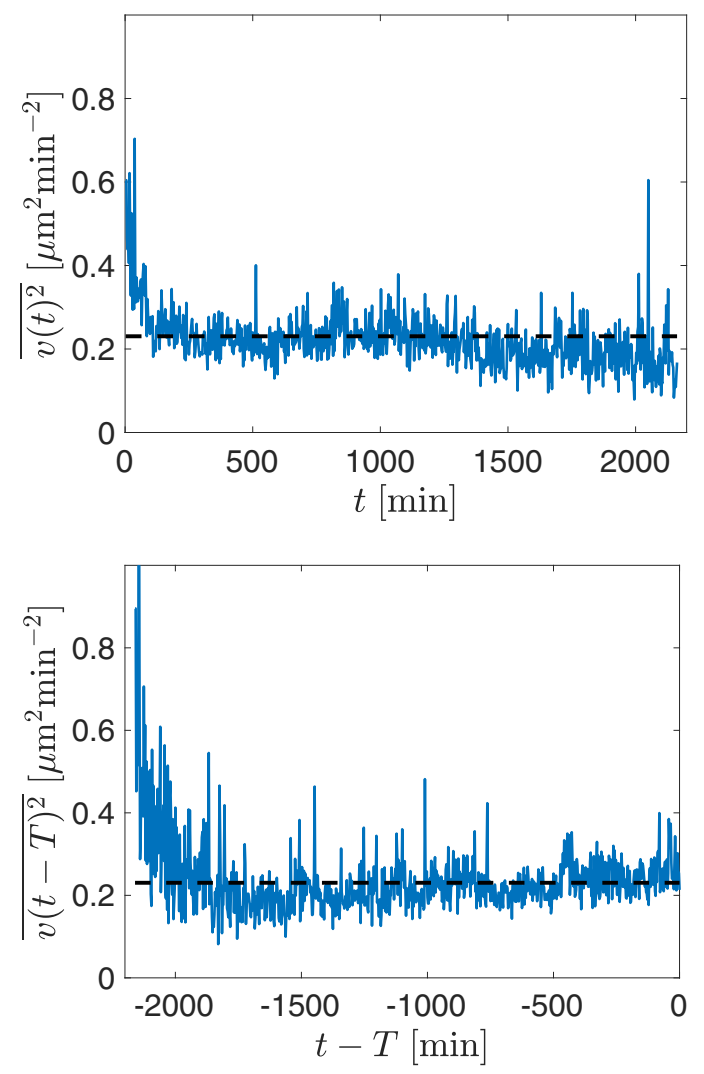

FIG. 5. Squared velocity averaged over all cells as a function of time. The horizontal black line indicates the average over all cells and over time.

\section{APPENDIX C: VELOCITY AUTOCORRELATION}

To derive the general formula (4) for the VACF in the frequency domain, we first note that

$$
\left\langle\tilde{F}_{R}(\omega) \tilde{F}_{R}\left(\omega^{\prime}\right)\right\rangle=2 \pi \delta\left(\omega+\omega^{\prime}\right) \Gamma_{R}(\omega),
$$

which follows from Eq. (2). The VACF can thus be written as

$$
\begin{aligned}
C_{v v}(t)= & \langle v(t) v(0)\rangle \\
= & \int_{-\infty}^{\infty} \frac{d \omega}{2 \pi} \int_{-\infty}^{\infty} \frac{d \omega^{\prime}}{2 \pi} e^{i \omega t} \\
& \times \frac{\left\langle\tilde{F}_{R}(\omega) \tilde{F}_{R}\left(\omega^{\prime}\right)\right\rangle}{\left[\tilde{\Gamma}_{v+}(\omega)+i \omega\right]\left[\tilde{\Gamma}_{v+}\left(\omega^{\prime}\right)+i \omega^{\prime}\right]} \\
= & \int_{-\infty}^{\infty} \frac{d \omega}{2 \pi} e^{i \omega t} \frac{\tilde{\Gamma}_{R}(\omega)}{\left[\tilde{\Gamma}_{v+}(\omega)+i \omega\right]\left[\tilde{\Gamma}_{v+}(-\omega)-i \omega\right]},
\end{aligned}
$$

which is just the inverse Fourier transform of Eq. (4).

The VACF for a general substitute model reads in the frequency domain

$$
\tilde{C}_{v v}(\omega)=\frac{B \tilde{\Gamma}(\omega)}{\left(\tilde{\Gamma}_{+}(\omega)+i \omega\right)\left(\tilde{\Gamma}_{+}(-\omega)-i \omega\right)}=\frac{B \tilde{\Gamma}(\omega)}{\left|\tilde{\Gamma}_{+}(\omega)+i \omega\right|^{2}}
$$

[see Eq. (6) in the main text]. Introducing the velocity response $\tilde{\chi}(\omega)=\left(i \omega+\tilde{\Gamma}_{+}(\omega)\right)^{-1}$, Eq. (C3) can be written as

$$
\tilde{C}_{v v}(\omega)=B \Gamma(\omega) \tilde{\chi}(\omega) \tilde{\chi}(-\omega)=B \tilde{\chi}(\omega)+B \tilde{\chi}(-\omega),
$$

where we used $\tilde{\Gamma}(\omega)=\tilde{\Gamma}_{+}(\omega)+\tilde{\Gamma}_{+}(-\omega)$. It follows that

$$
\begin{aligned}
C_{v v}(0) & =\int_{-\infty}^{\infty} \frac{d \omega}{2 \pi} \tilde{C}_{v v}(\omega)=B \int_{-\infty}^{\infty} \frac{d \omega}{2 \pi}[\tilde{\chi}(\omega)+\tilde{\chi}(-\omega)] \\
& =B \int_{-\infty}^{\infty} \frac{d \omega}{\pi} \tilde{\chi}(\omega) .
\end{aligned}
$$

Since the response function $\tilde{\chi}(\omega)$ is causal, it has no poles in the lower half complex plane. Closing the contour in the lower half plane leads to

$$
\begin{aligned}
C_{v v}(0) & =\frac{B}{\pi} \int_{-\infty}^{\infty} d \omega \tilde{\chi}(\omega)=-\frac{B}{\pi} \int_{\operatorname{arc}} d \omega \tilde{\chi}(\omega) \\
& =-\frac{B}{\pi} \lim _{R \rightarrow \infty} \int_{0}^{-\pi} d \varphi \frac{i R e^{i \varphi}}{i \operatorname{Re}^{i \varphi}+\tilde{\Gamma}_{+}\left(\operatorname{Re} e^{i \varphi}\right)} \\
& =-\frac{B}{\pi} \int_{0}^{-\pi} d \varphi=B
\end{aligned}
$$

where we assumed that $\left|\tilde{\Gamma}_{+}(z)\right|<\infty$ for all $z \in \mathbb{C}$.

\section{APPENDIX D: VELOCITY GREEN'S FUNCTION}

Since we model the cell velocity $v(t)$ as a stationary Gaussian process, the corresponding joint probability distribution, i.e., the non-normalized Green's function, is given by a bivariate normal distribution

$$
p\left(v_{2}, t_{2} ; v_{1}, t_{1}\right)=\frac{\exp \left(-\vec{v}^{T} \Sigma^{-1} \vec{v} / 2\right)}{2 \pi \sqrt{|\Sigma|}}
$$

Here, $\vec{v}^{T}=\left(v_{1}, v_{2}\right)$ and the covariance matrix is given by

$$
\begin{aligned}
\Sigma & =\left(\begin{array}{cc}
\left\langle v\left(t_{1}\right) v\left(t_{1}\right)\right\rangle & \left\langle v\left(t_{1}\right) v\left(t_{2}\right)\right\rangle \\
\left\langle v\left(t_{1}\right) v\left(t_{2}\right)\right\rangle & \left\langle v\left(t_{2}\right) v\left(t_{2}\right)\right\rangle
\end{array}\right) \\
& =\left(\begin{array}{cc}
C_{v v}(0) & C_{v v}\left(t_{2}-t_{1}\right) \\
C_{v v}\left(t_{2}-t_{1}\right) & C_{v v}(0)
\end{array}\right) .
\end{aligned}
$$

Since the velocity is normally distributed, $p(v)=$ $\exp \left[-v^{2} / 2 C_{v v}(0)\right] / \sqrt{2 \pi C_{v v}(0)}$, the normalized Green's function, which is the distribution of $v\left(t_{2}\right)=v_{2}$ conditional on $v\left(t_{1}\right)=v_{1}$, is given by

$$
\begin{gathered}
p\left(v_{2}, t_{2} \mid v_{1}, t_{1}\right)=\frac{p\left(v_{2}, t_{2} ; v_{1}, t_{1}\right)}{p\left(v_{1}, t_{1}\right)} \\
=\frac{\exp \left(-\frac{\left[v_{2}-v_{1} C_{v v}\left(t_{2}-t_{1}\right) / C_{v v}(0)\right]^{2}}{2 C_{v v}(0)\left[1-C_{v v}\left(t_{2}-t_{1}\right)^{2} / C_{v v}(0)^{2}\right]}\right)}{\sqrt{2 \pi C_{v v}(0)\left[1-C_{v v}\left(t_{1}-t_{2}\right)^{2} / C_{v v}(0)^{2}\right]}},
\end{gathered}
$$

from which the conditional expectation value of the velocity can be read off as

$$
\left.\left\langle v\left(t_{2}\right)\right\rangle\right|_{v\left(t_{1}\right)=v_{1}}=\frac{C_{v v}\left(t_{2}-t_{1}\right)}{C_{v v}(0)} v_{1} .
$$

Note that the Green's function can be entirely expressed in terms of the VACF. 


\section{APPENDIX E: ITERATIVE SOLUTION OF THE MEMORY EQUATION}

To extract the memory kernel from a discrete VACF, an appropriate discretization scheme for Eq. (7) needs to be chosen first. Note that since the memory kernel $\Gamma(t)$ is a symmetric function, its running integral $G(t)=\int_{0}^{t} d t^{\prime} \Gamma\left(t^{\prime}\right)$ is necessarily antisymmetric. We approximate the integral in Eq. (7) via the trapezoidal rule:

$$
\begin{aligned}
C_{v v}^{\text {expt }}(n \Delta)-C_{v v}^{\text {expt }}(0) & =-\frac{\Delta}{2} \sum_{i=0}^{n-1} G((n-i-1 / 2) \Delta)\left[C_{v v}^{\text {expt }}((i+1) \Delta)+C_{v v}^{\text {expt }}(i \Delta)\right] \Rightarrow \Delta G((n+1 / 2) \Delta) \\
& =-2 \frac{C_{v v}^{\text {expt }}((n+1) \Delta)-C_{v v}^{\text {expt }}(0)}{C_{v v}^{\text {expt }}(\Delta)+C_{v v}^{\text {expt }}(0)}-\sum_{i=1}^{n} \Delta G((n-i+1 / 2) \Delta) \frac{C_{v v}^{\text {expt }}((i+1) \Delta)+C_{v v}^{\text {expt }}(i \Delta)}{C_{v v}^{\text {expt }}(0)+C_{v v}^{\text {expt }}(\Delta)},
\end{aligned}
$$

where the sample points $G((n+1 / 2) \Delta)$ are located in-between the sample points of the discrete VACF $C_{v v}^{\text {expt }}(n \Delta)$. The discrete memory kernel $\Gamma(i \Delta)$ is then obtained via the central difference according to

$$
\Gamma(i \Delta)=\frac{G((i+1 / 2) \Delta)-G((i-1 / 2) \Delta)}{\Delta}, \quad \Rightarrow \quad \Gamma(0)=\frac{2 G(\Delta / 2)}{\Delta} .
$$

The expression for $\Gamma(0)$ follows using the antisymmetry of $G((i+1 / 2) \Delta)$.

\section{APPENDIX F: DISCRETE VELOCITY AUTOCORRELATION FUNCTION}

For a given finite-time resolution $\Delta$, the autocorrelation $C_{v v}^{\text {expt }}(j \Delta)$ of the discrete velocity $v(j \Delta)=[x(j \Delta+\Delta / 2]-$ $x[j \Delta-\Delta / 2)] / \Delta$ can be obtained from the continuous MSD by taking the second central difference. To see this, we first note that from the definition of the discrete velocity, it follows that

$$
x(j \Delta+\Delta / 2)=x(\Delta / 2)+\Delta \sum_{k=1}^{j} v(j \Delta) .
$$

The MSD for lag time $j \Delta$ can thus be written as

$$
\begin{aligned}
C_{\mathrm{MSD}}^{\mathrm{expt}}(j \Delta) & =\left\langle[x(j \Delta+\Delta / 2)-x(\Delta / 2)]^{2}\right\rangle \\
& =\left\langle\left(x(\Delta / 2)+\Delta \sum_{k=1}^{j} v(k \Delta)-x(\Delta / 2)\right)^{2}\right\rangle \\
& =\Delta^{2} \sum_{l, k=1}^{j}\langle v(l \Delta) v(k \Delta)\rangle \\
& =j \Delta^{2}\left\langle v(0)^{2}\right\rangle+2 \Delta^{2} \sum_{l=1}^{j} \sum_{k=1}^{l-1}\langle v((l-k) \Delta) v(0)\rangle .
\end{aligned}
$$

In the continuous case, the MSD and the VACF are related via $\frac{1}{2} \frac{d^{2}}{d t^{2}} C_{\mathrm{MSD}}(t)=C_{v v}(t)$, which carries over to the discrete case if one replaces the second derivative by the second central finite difference:

$$
\begin{aligned}
& \frac{C_{\mathrm{MSD}}^{\mathrm{expt}}((j+1) \Delta)-2 C_{\mathrm{MSD}}^{\mathrm{expt}}(j \Delta)+C_{\mathrm{MSD}}^{\mathrm{expt}}((j-1) \Delta)}{2 \Delta^{2}} \\
& \quad=\sum_{k=0}^{j-1}\langle v((j-k) \Delta) v(0)\rangle-\sum_{k=0}^{j-2}\langle v((i-k-1) \Delta) v(0)\rangle \\
& =\langle v(j \Delta) v(0)\rangle=C_{v v}^{\operatorname{expt}}(j \Delta) .
\end{aligned}
$$

An important special case is $\left\langle v(0)^{2}\right\rangle=C_{v v}^{\text {exp }}(0)$. Since the MSD is a symmetric function and zero at the origin, we have

$$
C_{v v}^{\mathrm{expt}}(0)=\frac{C_{\mathrm{MSD}}^{\mathrm{exp}}(\Delta)}{\Delta^{2}}
$$

As mentioned in the main text, the VACF of the experimental data was estimated by computing the autocorrelation function of the position increments $v(j \Delta)$ rather than by computing the finite difference of the MSD since the former method produces a less noisy VACF. Averages of the experimental VACF and MSD data over different cells are weighted with the individual trajectory lengths. Using Eq. (F3), the effect of localization noise with a deviation $\sigma_{\text {loc }}$ on the discrete VACF can be derived as

$$
\begin{aligned}
& C_{v v}^{\text {expt }}(0)=\frac{C_{\mathrm{MSD}}^{\text {expt }}(\Delta)}{\Delta^{2}}=\frac{C_{\mathrm{MSD}}(\Delta)+2 \sigma_{\mathrm{loc}}^{2}}{\Delta^{2}} \\
& =\hat{C}_{v v}^{\text {expt }}(0)+2 \frac{\sigma_{\text {loc }}^{2}}{\Delta^{2}}, \\
& C_{v v}^{\operatorname{expt}}(\Delta)=\frac{C_{\mathrm{MSD}}^{\mathrm{expt}}(2 \Delta)-2 C_{\mathrm{MSD}}^{\mathrm{expt}}(\Delta)}{2 \Delta^{2}} \\
& =\frac{C_{\mathrm{MSD}}(2 \Delta)+2 \sigma_{\mathrm{loc}}^{2}-2 C_{\mathrm{MSD}}(\Delta)-4 \sigma_{\mathrm{loc}}^{2}}{2 \Delta^{2}} \\
& =\hat{C}_{v v}^{\operatorname{expt}}(\Delta)-\frac{\sigma_{\mathrm{loc}}^{2}}{\Delta^{2}}, \\
& C_{v v}^{\operatorname{expt}}(j \Delta)=\hat{C}_{v v}^{\operatorname{expt}}(j \Delta), \quad j>1
\end{aligned}
$$

where $\hat{C}_{v v}^{\text {expt }}(j \Delta)$ denotes the discrete VACF in the absence of localization noise. As can be seen, the localization noise only affects the first two data points of the discrete VACF regardless of the sampling time $\Delta[17,43]$.

\section{APPENDIX G: DETAILS OF THE FITTING PROCEDURE}

Starting from a continuous model for the MSD (or equivalently the VACF) without localization noise, discretization effects as well as localization noise effects are accounted for in Eq. (16). The fits of Eq. (16) to experimental data are performed using MATLAB 2016b (Mathworks). We employ a built in implementation of the trust region method to perform 
the nonlinear least-square fit of Eq. (16) to the first $500 \mathrm{~min}$ of the cell-averaged VACF. The root-mean-square deviation (RMSD) defined by

$$
r^{2}=\frac{1}{j_{\max }} \sum_{j=0}^{j_{\max }-1}\left[C_{v v}^{\operatorname{expt}}(j \Delta)-C_{v v}^{\mathrm{fit}}(j \Delta)\right]^{2}
$$

between the model prediction $C_{v v}^{\text {expt }}$ and the experimental data $C_{v v}^{\text {data }}$ is $r \approx 0.0015$. We obtain the following values for the fit parameters:

$$
\begin{aligned}
a & =0.15 \mathrm{~min}^{-1}, \\
b & =-0.023 \mathrm{~min}^{-2}, \\
\tau_{m} & =6.02 \mathrm{~min}, \\
B & =0.14 \mu \mathrm{m}^{2} \mathrm{~min}^{-2}, \\
\sigma_{\mathrm{loc}} & =0.53 \mu \mathrm{m} .
\end{aligned}
$$

These values give the timescales $\tau_{b}=3.23 \mathrm{~min}$ and $\tau_{p}=$ $164 \mathrm{~min}$. The corresponding estimate for the diffusion constant (12) is given by $D=12.7 \mu \mathrm{m}^{2} \mathrm{~min}^{-1}$ and only slightly deviates from $D^{\text {expt }}=12.3 \mu \mathrm{m}^{2} \mathrm{~min}^{-1}$ obtained via integrating the first 500 mins of the experimental VACF.

For the single-cell VACFs, Eq. (16) is fitted to the experimental data from $t=0$ to half the temporal length of the trajectory. The fits are performed for four different starting values

\begin{tabular}{c|c|c|c|c} 
& No. 1 & No. 2 & No. 3 & No. 4 \\
\hline$a$ & $*$ & $*$ & $*$ & $*$ \\
\hline$b$ & $*$ & $*$ & 0 & 0 \\
\hline$\tau_{m}$ & $*$ & $*$ & $*$ & $*$ \\
\hline$B$ & $*$ & $*$ & $*$ & $*$ \\
\hline$\sigma_{\mathrm{loc}}$ & $*$ & 0 & $*$ & 0
\end{tabular}

where the asterisk denotes the corresponding parameter values obtained from the fit to the cell-averaged VACF. For the fitting procedure, we restrict the parameter values to lie within the bounds

$$
\begin{aligned}
0 & <a<10 \mathrm{~min}^{-1}, \\
-10 & <b<10 \mathrm{~min}^{-2}, \\
0.01 & <\tau_{m}<10^{5} \mathrm{~min}, \\
0.01 & <B<10^{3} \mu \mathrm{m}^{2} \mathrm{~min}^{-2}, \\
0 & <\sigma_{\mathrm{loc}}<10 \mu \mathrm{m} .
\end{aligned}
$$

The average RMSD of the best single-cell fits is $r \approx 0.022$.

For the diffusion constant we obtain $D^{\text {expt }}=$ $12.3 \mu \mathrm{m}^{2} \mathrm{~min}^{-1}$ via integrating the first $500 \mathrm{~min}$ of the experimental cell-averaged VACF with the trapezoidal rule according to

$$
D^{\text {expt }}=\frac{\Delta}{2} C_{v v}^{\text {expt }}(0)+\Delta \sum_{j=1}^{j_{\max }-1} C_{v v}^{\text {expt }}(j \Delta)+\frac{\Delta}{2} C_{v v}^{\text {expt }}\left(j_{\max } \Delta\right) .
$$

Note that $D^{\text {expt }}$ is independent of the localization uncertainty $\sigma_{\text {loc }}$ (cf. Appendix F). To estimate the uncertainty of $D_{\text {expt }}$, we first need an estimate of the uncertainty of the VACF. Estimating the uncertainty of an autocorrelation function via
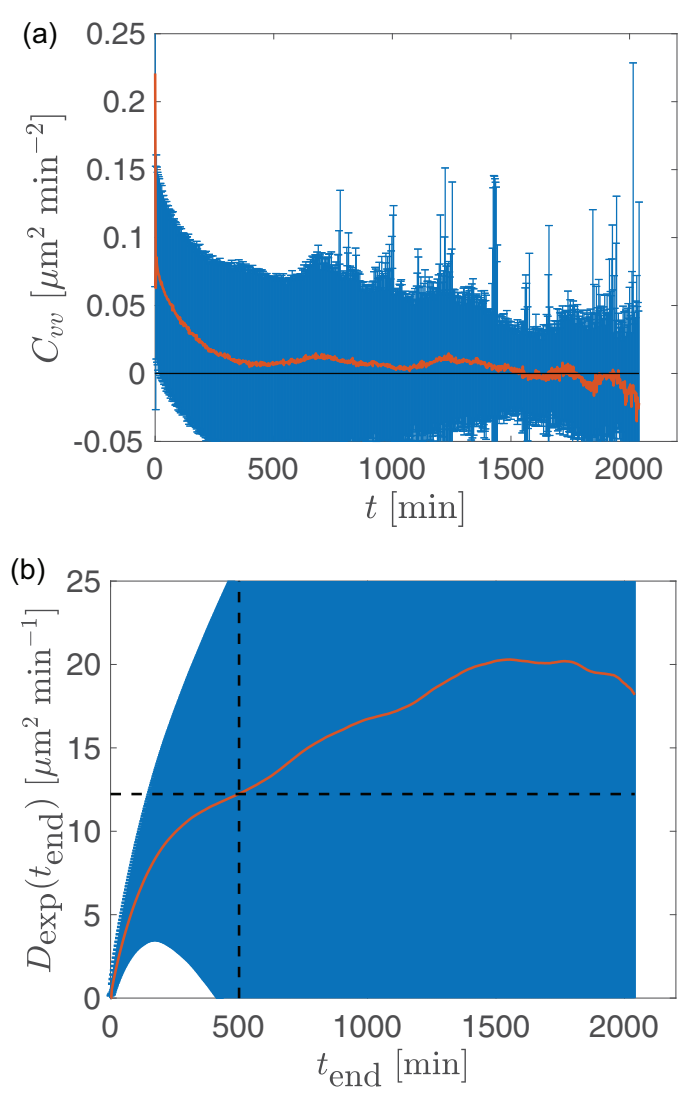

FIG. 6. (a) Cell-averaged VACF (solid red line). The error bars denote the root-mean-square deviation as obtained by comparing the cell-averaged VACF with the single-cell VACFs (see text). (b) Running integral (solid red line) over the cell-averaged VACF, used to estimate the diffusion constant $D_{\text {expt }}$. Error bars (in blue) are obtained by taking the running integral of the RMSD of the VACF.

the standard error underestimates the true uncertainty since the data are correlated. Instead, we exploit the fact that we are averaging over many different cells and estimate the error from the scattering of the single-cell VACFs around the cellaveraged VACF by calculating the root-mean-square deviation (RMSD) acording to

$$
\operatorname{RMSD}(j \Delta)=\sqrt{\frac{1}{n(j \Delta)} \sum_{i}\left[C_{v v}^{\mathrm{expt}}(j \Delta)-C_{v v}^{\mathrm{SC}, \mathrm{i}}(j \Delta)\right]^{2}} .
$$

Here, $C_{v v}^{\mathrm{SC}, \mathrm{i}}$ denotes the experimental VACF of the $i$ th single cell, $n(j \Delta)$ denotes the number of trajectories of at least length $j \Delta$, and the sum is taken over all trajectories which are at least of length $n(j \Delta)$. Figure 6(a) shows the cellaveraged VACF together with error bars denoting the RMSD. The resulting running integral (i.e., the estimate of $D_{\text {expt }}$ ) is shown in Fig. 6(b), where the error bars denote the estimate of the maximal uncertainty that follows from integrating the RMSD, Eq. (G4), of the VACF. The vertical black dashed line indicates the upper integration limit $t_{\mathrm{end}}=500 \mathrm{~min}$, which is the value we used in our analysis. This choice for the upper integration limit is rationalized by the observation that beyond $500 \mathrm{~min}$ the noise of the the cell-averaged VACF by far exceeds the value of the VACF itself, as shown in Fig. 6(a). 
(a)

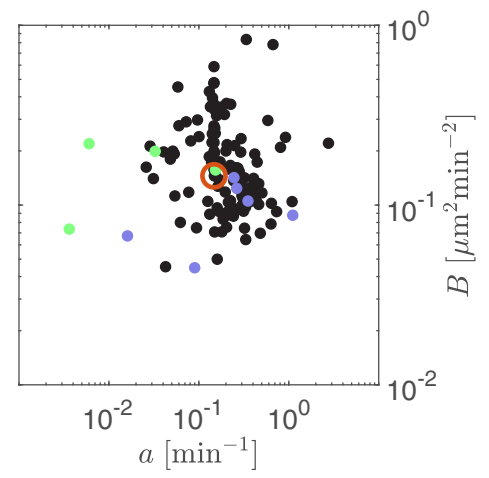

(d)

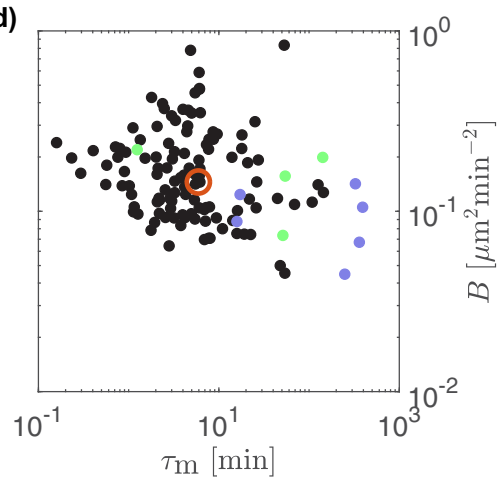

(g)

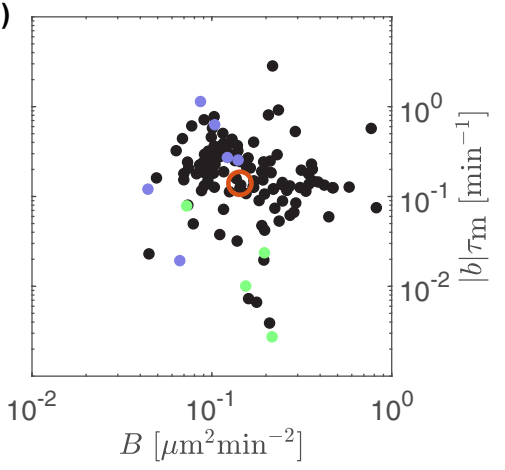

(b)

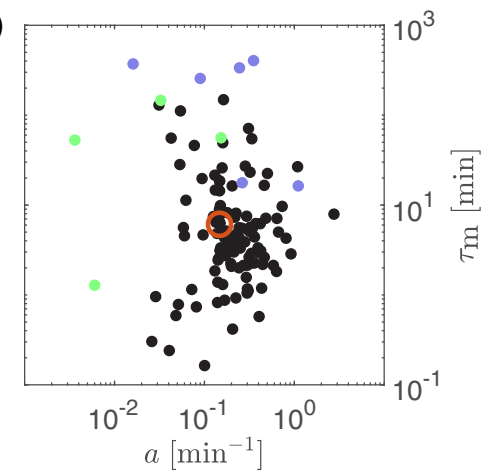

(e)

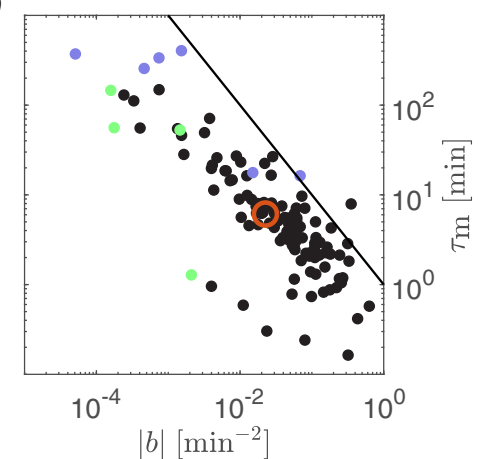

(h)

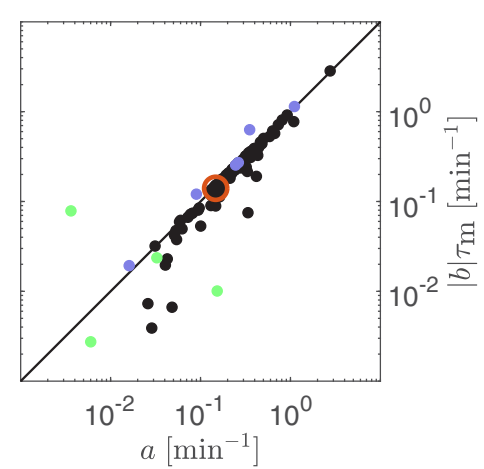

(c)

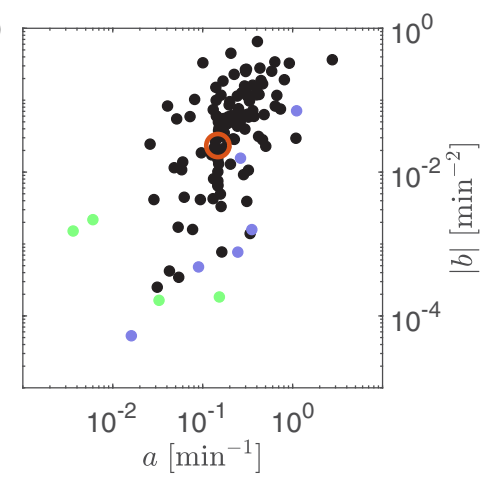

(f)

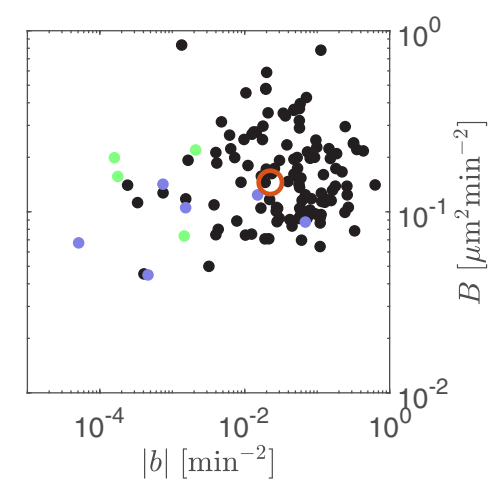

FIG. 7. Correlation analysis of single-cell kernel parameters $a, b, \tau_{m}, B$ obtained from fits of Eq. (16) to single-cell VACFs. Cell data for which $b$ is positive are shown as green circles, blue circles denote cells for which $D$ according to Eq. (12) is negative, black circles denote the majority of cells for which $b$ is negative and $D$ positive. Red open circles denote the result from the analysis of the cell-averaged VACF (cf. Fig. 3 in the main text). The straight, solid black lines in (e) and (h) denote (inverse) linear scaling.

\section{APPENDIX H: CORRELATION ANALYSIS OF SINGLE-CELL PARAMETERS}

Here, we provide the full correlation analysis between the kernel parameters $a, b, \tau_{m}, B$ and the integrated exponential tail $b \tau_{m}$, extracted from single-cell trajectories (see Fig. 7). Aside from the (inverse) correlations between $b$ and $\tau_{m}$ and between $a$ and $b \tau_{m}$ discussed in the main text, no further correlations are discernible. Notably, the kernel amplitudes $a$ and $b$ are rather uncorrelated [see Fig. 7(c)].

Figure 8 shows the correlation analysis for the equivalent set of parameters $D, B, \tau_{b}, \tau_{p}$ which correspond to distinct features in the MSD and the VACF. Aside from the correlation between $\tau_{p}$ and $D$ discussed in the main text, no other correlations are discernible.

In all cases, the data do not fall into distinct clusters and the single-cell parameters scatter around the values extracted from the cell-averaged VACF, which are denoted by red circles.

\section{APPENDIX I: EQUILIBRIUM MARKOVIAN MODEL}

We discuss how a memory kernel with a negative exponential tail arises from a Markovian system of coupled particles, which satisfies the fluctuation-dissipation theorem and thus is in equilibrium. We consider a system of two particles, whose velocities are coupled by a generalized friction matrix,

$$
\begin{aligned}
& \dot{v}_{1}(t)=-\gamma_{1} v_{1}(t)-\gamma_{12} v_{2}(t)+\phi_{1} \xi_{1}(t)+\phi_{12} \xi_{2}(t), \\
& \dot{v}_{2}(t)=-\gamma_{2} v_{2}(t)-\gamma_{12} v_{1}(t)+\phi_{2} \xi_{2}(t)+\phi_{12} \xi_{1}(t) .
\end{aligned}
$$

Here, $\xi_{i}(t)$ are two independent realizations of white, Gaussian noise, i.e., $\left\langle\xi_{i}(t) \xi_{j}\left(t^{\prime}\right)\right\rangle=2 \delta_{i j} \delta\left(t-t^{\prime}\right)$. The goal is to construct an effective, non-Markovian dynamics for the first 
(a)

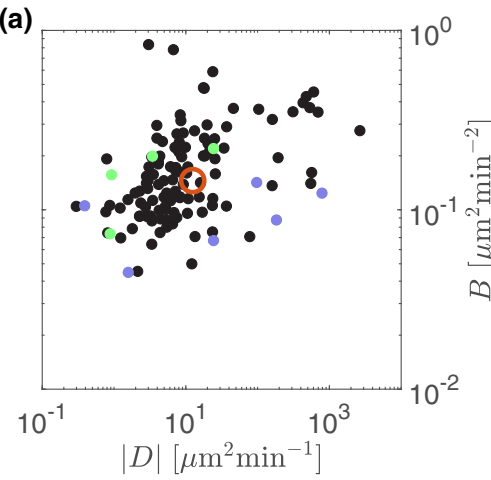

(d)

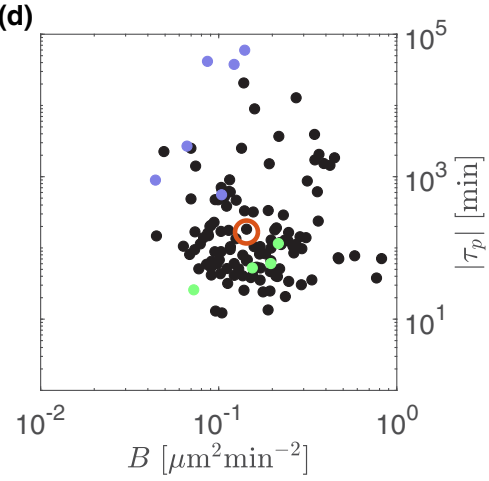

(b)

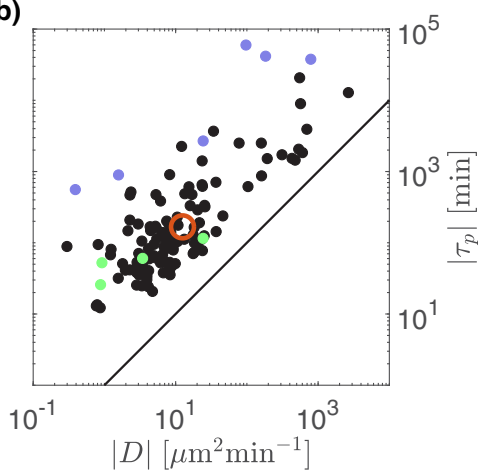

(e)

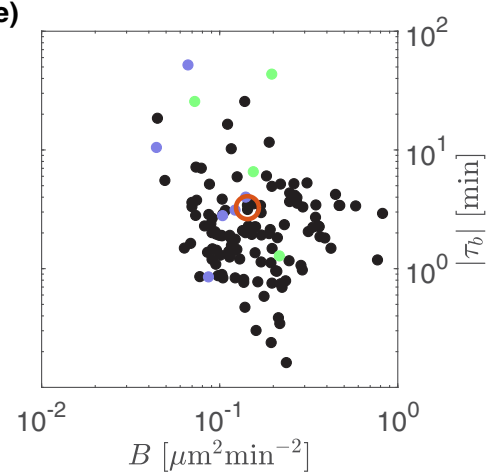

(c)

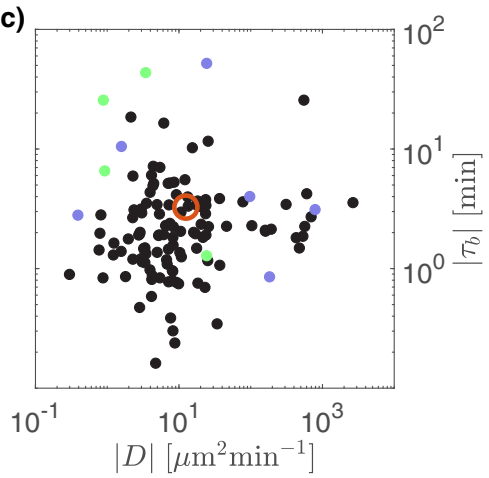

(f)

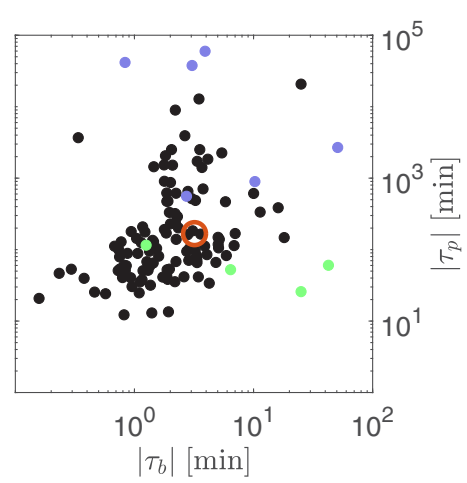

FIG. 8. Correlation analysis of the parameter set $D, B, \tau_{b}, \tau_{p}$. The color code for the data points is the same as in Fig. 7. The solid black line in (b) illustrates the linear scaling between the diffusion constant $D$ and the persistence time $\tau_{p}$. Aside from that, no other correlations are discernible.

particle by integrating out the contribution of the second particle. We obtain

$v_{2}(t)=\int_{-\infty}^{t} d t^{\prime} e^{-\left(t-t^{\prime}\right) \gamma_{2}}\left[-\gamma_{12} v_{1}\left(t^{\prime}\right)+\phi_{2} \xi_{2}\left(t^{\prime}\right)+\phi_{12} \xi_{1}\left(t^{\prime}\right)\right]$.

Inserting this solution into the equation of motion of the first particle, we obtain

$$
\dot{v}_{1}(t)=-\int_{-\infty}^{t} d t^{\prime} \Gamma\left(t-t^{\prime}\right) v_{1}\left(t^{\prime}\right)+F_{R}(t),
$$

with a memory kernel

$$
\Gamma(t)=2 \gamma_{1} \delta(t)-\gamma_{12}^{2} e^{-|t| \gamma_{2}} .
$$

It can be seen that the coupling between velocities, mediated by the friction coupling coefficient $\gamma_{12}$, leads to a negative exponential tail in the memory kernel. The effective random force acting on the first particle is given by

$$
\begin{aligned}
F_{R}(t)= & \phi_{1} \xi_{1}(t)+\phi_{12} \xi_{2}(t) \\
& -\int_{-\infty}^{t} d t^{\prime} e^{-\left(t-t^{\prime}\right) \gamma_{2}}\left[\gamma_{12} \phi_{2} \xi_{2}\left(t^{\prime}\right)+\gamma_{12} \phi_{12} \xi_{1}\left(t^{\prime}\right)\right] .
\end{aligned}
$$

Thus, we obtain the following expression for the autocorrelation function of the random force:

$$
\begin{aligned}
\left\langle F_{R}(t) F_{R}(0)\right\rangle=\Gamma_{R}(t)= & 2\left(\phi_{1}^{2}+\phi_{12}^{2}\right) \delta(t) \\
& -\left[2 \gamma_{12}\left(\phi_{2} \phi_{12}+\phi_{1} \phi_{12}\right)\right. \\
& \left.-\gamma_{12}^{2}\left(\phi_{2}^{2}+\phi_{12}^{2}\right) / \gamma_{2}\right] e^{-|t| \gamma_{2}} .
\end{aligned}
$$

The fluctuation-dissipation theorem, i.e., the relation $\Gamma_{R}(t)=$ $B \Gamma(t)$, holds if the following conditions are satisfied:

$$
\begin{aligned}
B \gamma_{1} & =\phi_{1}^{2}+\phi_{12}^{2}, \\
B \gamma_{2} & =\phi_{2}^{2}+\phi_{12}^{2}, \\
B \gamma_{12} & =\phi_{12}\left(\phi_{1}+\phi_{2}\right) .
\end{aligned}
$$

The connection to the kernel of the substitute model (8) in the main text is established by comparison with Eq. (I4). This leads to the following mapping:

$$
\begin{gathered}
a=\gamma_{1}, \\
b=-\gamma_{12}^{2}, \\
\tau_{m}=\gamma_{2}^{-1} .
\end{gathered}
$$

\section{APPENDIX J: NONEQUILIBRIUM MARKOVIAN MODELS}

The closed form expression for the VACF of the substitute model (8) reads as

$$
\tilde{C}_{v v}(\omega)=2 B \frac{a \tau_{m}^{2} \omega^{2}+a+b \tau_{m}}{\omega^{4} \tau_{m}^{2}+\omega^{2}\left(1-2 \tau_{m}^{2} b+a^{2} \tau_{m}^{2}\right)+\left(a+b \tau_{m}\right)^{2}} .
$$

This expression has poles at $\omega= \pm i / \tau_{p}$ and $\pm i / \tau_{b}$, which are given explicitly in Eq. (10) in the main text. Our goal is to 
find a nonequilibrium model that reproduces the above VACF. First, we note that

$$
\begin{aligned}
D=\frac{\tilde{C}_{v v}(0)}{2} & =\frac{B}{a+\tau_{m} b}, \\
\frac{1}{2} \lim _{\omega \rightarrow \infty} \omega^{2} \tilde{C}_{v v}(\omega) & =B a .
\end{aligned}
$$

As a starting point, we consider the following nonequilibrium model:

$$
\Gamma_{v}(t)=2 a_{v} \delta(t)+\frac{b_{v}}{\tau_{v}} e^{-t / \tau_{v}}, \quad \Gamma_{R}(t)=2 a_{R}^{2} \delta(t)+\frac{b_{R}^{2}}{\tau_{R}} e^{-t / \tau_{R}},
$$

whose associated VACF is given by

$$
\tilde{C}_{v v}(\omega)=\frac{2\left(1+\omega^{2} \tau_{v}^{2}\right)\left(a_{R}^{2}+b_{R}^{2}+a_{R}^{2} \tau_{R}^{2} \omega^{2}\right)}{\left(1+\omega^{2} \tau_{R}^{2}\right)\left[\omega^{2} \tau_{v}-i \omega\left(1+a_{v} \tau_{v}\right)-a_{v}-b_{v}\right]\left[\omega^{2} \tau_{v}+i \omega\left(1+a_{v} \tau_{v}\right)-a_{v}-b_{v}\right]} .
$$

By comparing Eqs. (J1) and (J4), we note that the powers of $\omega$ in the numerators and denominators do not match in general. There are two special cases of the model $(\mathrm{J} 3)$ which reproduce the correct functional form of Eq. (J1), these are obtained by setting either $b_{v}$ or $b_{R}$ to zero. We in the following consider these two cases separately.

Model I with $\mathbf{b}_{\mathbf{v}}=\mathbf{0}$. In this case the expression (J4) reduces to

$$
\tilde{C}_{v v}(\omega)=\frac{2\left(a_{R}^{2}+b_{R}^{2}+a_{R}^{2} \tau_{R}^{2} \omega^{2}\right)}{\left(1+\omega^{2} \tau_{R}^{2}\right)\left(-i \omega-a_{v}\right)\left(i \omega-a_{v}\right)} .
$$

Comparing with Eq. $(\mathrm{J} 2)$, we obtain the following relations:

$$
\begin{aligned}
\frac{a_{R}^{2}+b_{R}^{2}}{a_{v}^{2}} & =\frac{B}{a+\tau_{m} b}, \\
a_{R}^{2} & =B a .
\end{aligned}
$$

The VACF (J5) has poles $\omega= \pm i a_{v}$ and $\pm i / \tau_{R}$, which must coincide with the poles at $\omega= \pm i / \tau_{p}$ and $\pm i / \tau_{b}$. With the choice $a_{v}=1 / \tau_{b}$ and $\tau_{R}=\tau_{p}$ we obtain the mapping

$$
\begin{aligned}
a_{v} & =\frac{1}{\tau_{b}}, \\
\tau_{R} & =\tau_{p}, \\
a_{R}^{2} & =B a, \\
b_{R}^{2} & =\left(\frac{\tau_{p}}{\tau_{b} \tau_{m}}-a\right) B,
\end{aligned}
$$

as described in the main text, where we have used the relation $\tau_{p} \tau_{b} / \tau_{m}=1 /\left(a+b \tau_{m}\right)$.

The dynamics of this model can be described by two coupled Markovian equations of motion

$$
\begin{aligned}
& \dot{\eta}(t)=-\tau_{R}^{-1} \eta(t)+\tau_{R}^{-1} \xi_{1}(t), \\
& \dot{v}(t)=-a_{v} v(t)+a_{R} \xi_{0}(t)+b_{R} \eta(t),
\end{aligned}
$$

where $\eta(t)$ is a hidden fluctuating degree of freedom. In the equations, two white Gaussian noise variables $\xi_{i}(t)$ appear with correlators $\left\langle\xi_{i}(t) \xi_{j}\left(t^{\prime}\right)\right\rangle=2 \delta_{i j} \delta\left(t-t^{\prime}\right)$. With the alternative choice $a_{v}=1 / \tau_{p}$ and $\tau_{R}=\tau_{b}$ instead, we obtain $b_{R}^{2}<0$ and thus an imaginary coupling constant in Eq. (J8), which is unphysical and therefore discarded.

The effective random force $F_{R}(t)=a_{R} \xi_{0}(t)+b_{R} \eta(t)$ reproduces the desired correlation

$$
\left\langle F_{R}(t) F_{R}(0)\right\rangle=2 a_{R}^{2} \delta(t)+b_{R}^{2} e^{-t / \tau_{R}} / \tau_{R}
$$

To see this, we first note that the solution for $\eta(t)$ is given by

$$
\eta(t)=\frac{1}{\tau_{R}} \int_{-\infty}^{t} d t^{\prime} e^{-\left(t-t^{\prime}\right) / \tau_{R}} \xi_{1}(t) .
$$

The autocorrelation for $\eta(t)$ is thus given by

$$
\begin{aligned}
\langle\eta(t) \eta(0)\rangle & =\frac{2}{\tau_{R}^{2}} e^{-t / \tau_{R}} \int_{-\infty}^{0} d t^{\prime} e^{t^{\prime} / \tau_{R}} \int_{-\infty}^{t} d t^{\prime \prime} e^{t^{\prime \prime} / \tau_{R}} \delta\left(t^{\prime \prime}-t^{\prime}\right) \\
& =\frac{2}{\tau_{R}^{2}} e^{-t / \tau_{R}} \int_{-\infty}^{0} d t^{\prime} e^{2 t^{\prime} \tau_{R}}=\frac{1}{\tau_{R}} e^{-t / \tau_{R}} .
\end{aligned}
$$

Since $\xi_{0}(t)$ and $\xi_{1}(t)$ are uncorrelated, we have $\left\langle\eta(t) \xi_{0}\left(t^{\prime}\right)\right\rangle=$ 0 and Eq. (J9) follows.

Model II with $\mathbf{b}_{\mathbf{R}}=\mathbf{0}$. In this case the expression (J4) reduces to

$$
\tilde{C}_{v v}(\omega)=\frac{2 a_{R}^{2}\left(1+\tau_{v}^{2} \omega^{2}\right)}{\tau_{v}^{2}\left|\omega^{2}-i \omega\left(\tau_{v}^{-1}+a_{v}\right)-\tau_{v}^{-1}\left(a_{v}+b_{v}\right)\right|^{2}}
$$

We proceed in a similar fashion as for the $b_{v}=0$ case, the comparison with Eq. (J2) gives

$$
\begin{aligned}
\frac{a_{R}^{2}}{\left(a_{v}+b_{v}\right)^{2}} & =\frac{B}{a+\tau_{m} b}, \\
a_{R}^{2} & =B a .
\end{aligned}
$$

Equation (J12) has poles located at

$$
\omega_{ \pm}=i \frac{\tau_{v}^{-1}+a_{v}}{2} \pm \sqrt{-\frac{\left(\tau_{v}^{-1}+a_{v}\right)^{2}}{4}+\frac{a_{v}+b_{v}}{\tau_{v}}} .
$$

We consider the expressions for $\omega_{+}+\omega_{-}$and $\omega_{+} \omega_{-}$, which must coincide with $i / \tau_{b}+i / \tau_{p}$ and $-1 / \tau_{b} \tau_{b}$, respectively. This gives the conditions

$$
\begin{aligned}
\tau_{v}^{-1}+a_{v} & =\tau_{m}^{-1}+a, \\
\left(a_{v}+b_{v}\right) / \tau_{v} & =a \tau_{m}^{-1}+b .
\end{aligned}
$$

Solving explicitly for the parameters of the nonequilibrium model gives the following mapping:

$$
\begin{aligned}
& a_{v}=\tau_{m}^{-1}+a-\sqrt{\frac{a \tau_{m}^{-1}+b}{a \tau_{m}}} \\
& b_{v}=\sqrt{a \tau_{m}^{-1}+b}\left(\sqrt{a \tau_{m}}+\frac{1}{\sqrt{a \tau_{m}}}\right)-a-\tau_{m}^{-1},
\end{aligned}
$$




$$
\begin{aligned}
\tau_{v} & =\sqrt{\frac{a \tau_{m}^{2}}{a+b \tau_{m}},} \\
a_{R}^{2} & =B a,
\end{aligned}
$$

where the timescale $\tau_{v}$ was chosen to be positive. The friction kernel $\Gamma_{v}(t)$ is composed of a positive delta peak at the origin proportional to $a_{v}=0.27 \mathrm{~min}^{-1}$, followed by a negative tail proportional to $b_{v}=-0.229 \mathrm{~min}^{-1}$. The dynamics can be described by the following system of two coupled degrees of freedom:

$$
\begin{aligned}
& \dot{u}(t)=\tau_{v}^{-1} v(t)-\tau_{v}^{-1} u(t) \\
& \dot{v}(t)=-a_{v} v(t)-b_{v} u(t)+a_{R} \xi_{0}(t)
\end{aligned}
$$

These equations of motion can in fact be derived from the Hamiltonian

$$
H=\frac{1}{2}(u-v)^{2}-\frac{1}{2}\left(\frac{a_{v}}{b_{v}}+1\right) v^{2} .
$$

Note that since $a_{v} / b_{v}<-1$, the Hamiltonian is non-negative for all $u$ and $v$ and thus describes a system with a stable stationary state. For the conjugated variables $\tau_{v} \dot{u}(t)$ and $-b_{v}^{-1} \dot{v}(t)$, we obtain the equations of motion

$$
\begin{aligned}
\tau_{v} \dot{u}(t) & =v(t)-u(t)=-\frac{\partial H}{\partial u} \\
-b_{v}^{-1} \dot{v}(t) & =\frac{a_{v}}{b_{v}} v(t)+u(t)-\frac{a_{R}}{b_{v}} \xi_{0}(t) \\
& =-\frac{\partial H}{\partial v}-\frac{a_{R}}{b_{v}} \xi_{0}(t)
\end{aligned}
$$

which are identical to Eqs. (J17).
[1] A. J. Ridley, M. A. Schwartz, K. Burridge, R. A. Firtel, M. H Ginsberg, G. Borisy, J. T. Parsons, and A. R. Horwitz, Science 302, 1704 (2003).

[2] D. Selmeczi, L. Li, L. I. Pedersen, S. F. Nørrelykke, P. H. Hagedorn, S. Mosler, N. B. Larsen, E. C. Cox, and H. Flyvbjerg, Eur. Phys. J.: Spec. Top. 157, 1 (2008).

[3] P. Friedl and D. Gilmour, Nat. Rev. Mol. Cell Biol. 10, 445 (2009).

[4] C.-M. Svensson, A. Medyukhina, I. Belyaev, N. Al-Zaben, and M. T. Figge, Cytometry Part A 93, 357 (2017).

[5] R. Fürth, Z. Phys. 2, 244 (1920).

[6] M. H. Gail and C. W. Boone, Biophyis. J. 10, 980 (1970).

[7] G. A. Dunn and A. F. Brown, J. Cell Sci. Suppl. 8, 81 (1987).

[8] E. A. Codling, M. J. Plank, and S. Benhamou, J. R. Soc. Interface 5, 813 (2008).

[9] D. Selmeczi, S. Mosler, P. H. Hagedorn, N. B. Larsen, and H. Flyvbjerg, Biophys. J. 89, 912 (2005).

[10] L. Li, S. F. Nørrelykke, and E. C. Cox, PLoS One 3, e2093 (2008).

[11] H. Takagi, M. J. Sato, T. Yanagida, and M. Ueda, PLoS One 3, e2648 (2008).

[12] L. Li, E. C. Cox, and H. Flyvbjerg, Phys. Biol. 8, 046006 (2011).

[13] O. Pohl, M. Hintsche, Z. Alirezaeizanjani, M. Seyrich, C. Beta, and H. Stark, PLoS Comput. Biol. 13, e1005329 (2017).

[14] H. U. Bödeker, C. Beta, T. D. Frank, and E. Bodenschatz, Europhys. Lett. 90, 28005 (2010).

[15] G. Amselem, M. Theves, A. Bae, E. Bodenschatz, and C. Beta, PLoS One 7, e37213 (2012).

[16] D. B. Brückner, A. Fink, C. Schreiber, P. J. F. Röttgermann, J. O. Rädler, and C. P. Broedersz, Nat. Phys. 15, 595 (2019).

[17] P.-H. Wu, A. Giri, S. X. Sun, and D. Wirtz, Proc. Natl. Acad. Sci. USA 111, 3949 (2014).

[18] P. Dieterich, R. Klages, R. Preuss, and A. Schwab, Proc. Natl. Acad. Sci. USA 105, 459 (2008).

[19] T. H. Harris, E. J. Banigan, D. A. Christian, C. Konradt, E. D. Tait Wojno, K. Norose, E. H. Wilson, B. John, W. Weninger, A. D. Luster, A. J. Liu, and C. A. Hunter, Nature (London) 486, 545 (2012).
[20] P. Romanczuk, M. Romensky, D. Scholz, V. Lobaskin, and L. Schimansky-Geier, Eur. Phys. J.: Spec. Top. 224, 1215 (2015).

[21] R. Zwanzig, Phys. Rev. 124, 983 (1961).

[22] H. Mori, Prog. Theor. Phys. 33, 423 (1965).

[23] R. Zwanzig, Nonequilibrium Statistical Mechanics (Oxford University Press, Oxford, 2001).

[24] D. Lesnicki, R. Vuilleumier, A. Carof, and B. Rotenberg, Phys. Rev. Lett. 116, 147804 (2016).

[25] J. O. Daldrop, B. G. Kowalik, and R. R. Netz, Phys. Rev. X 7, 041065 (2017).

[26] J. O. Daldrop, J. Kappler, F. N. Brünig, and R. R. Netz, Proc. Natl. Acad. Sci. USA 115, 5169 (2018).

[27] T. G. Mason and D. A. Weitz, Phys. Rev. Lett. 74, 1250 (1995).

[28] T. Indei, J. D. Schieber, A. Córdoba, and E. Pilyugina, Phys. Rev. E 85, 021504 (2012).

[29] O. F. Lange and H. Grubmüller, J. Chem. Phys. 124, 214903 (2006).

[30] D. T. Schmitt and M. Schulz, Phys. Rev. E 73, 056204 (2006).

[31] M. Niemann, T. Laubrich, E. Olbrich, and H. Kantz, Phys. Rev. E 77, 011117 (2008).

[32] J. Kappler, J. O. Daldrop, F. N. Brünig, M. D. Boehle, and R. R. Netz, J. Chem. Phys. 148, 014903 (2018).

[33] J. Kappler, V. B. Hinrichsen, and R. R. Netz, Eur. Phys. J. E 42, 119 (2019).

[34] A. D. Doyle, F. W. Wang, K. Matsumoto, and K. M. Yamada, J. Cell Biol. 184, 481 (2009).

[35] P. Maiuri, E. Terriac, P. Paul-Gilloteaux, T. Vignaud, K. McNally, J. Onuffer, K. Thorn, P. A. Nguyen, N. Georgoulia, D. Soong, A. Jayo, N. Beil, J. Beneke, J. C. H. Lim, C. P. Y. Sim, Y. S. Chu, A. Jimenez-Dalmaroni, J. F. Joanny, J. P. Thiery, H. Erfle et al., Curr Biol. 22, R673 (2012).

[36] C. Schreiber, F. J. Segerer, E. Wagner, A. Roidl, and J. O. Rädler, Sci. Rep. 6, 26858 (2016).

[37] V. Belaud, T. Petithory, A. Ponche, C. Mauclair, C. Donnet, L. Pieuchot, S. Benayoun, and K. Anselme, Biointerphases 13, 06D408 (2018).

[38] C. Metzner, C. Mark, J. Steinwachs, L. Lautscham, F. Stadler, and B. Fabry, Nat. Commun. 6, 7516 (2015). 
[39] Y. Matsuda, I. Hanasaki, R. Iwao, H. Yamaguchi, and T. Niimi, Phys. Chem. Chem. Phys. 20, 24099 (2018).

[40] M. Hinczewski, Y. von Hansen, J. Dzubiella, and R. R. Netz, J. Chem. Phys. 132, 245103 (2010).

[41] B. J. Berne and G. D. Harp, On the calculation of time correlation functions, Advances in Chemical Physics (WileyBlackwell, Hoboken, NJ, 2007), pp. 63-227.

[42] B. Kowalik, J. O. Daldrop, J. Kappler, J. C. F. Schulz, A. Schlaich, and R. R. Netz, Phys. Rev. E 100, 012126 (2019).

[43] J. N. Pedersen, L. Li, C. Gradinaru, R. H. Austin, E. C. Cox, and H. Flyvbjerg, Phys. Rev. E 94, 062401 (2016).

[44] P. Maiuri, J. F. Rupprecht, S. Wieser, V. Ruprecht, O. Benichou, N. Carpi, M. Coppey, S. De Beco, N. Gov, C. P. Heisenberg, C. Lage Crespo, F. Lautenschlaeger, M. Le Berre, A. M. LennonDumenil, M. Raab, H. R. Thiam, M. Piel, M. Michael Sixt, and R. I. Voituriez, Cell 161, 374 (2015).
[45] F. Peruani and L. G. Morelli, Phys. Rev. Lett. 99, 010602 (2007).

[46] S. Shankar and M. C. Marchetti, Phys. Rev. E 98, 020604(R) (2018).

[47] Y. Fily and M. C. Marchetti, Phys. Rev. Lett. 108, 235702 (2012).

[48] T. F. F. Farage, P. Krinninger, and J. M. Brader, Phys. Rev. E 91, 042310 (2015).

[49] C. Battle, C. P. Broedersz, N. Fakhri, V. F. Geyer, J. Howard, C. F. Schmidt, and F. C. MacKintosh, Science 352, 604 (2016).

[50] R. J. Petrie, A. D. Doyle, and K. M. Yamada, Nat. Rev. Mol. Cell Biol. 10, 538 (2009).

[51] J. Zimmermann and M. Falcke, PloS One 9, e87638 (2014).

[52] F. Raynaud, M. E. Ambühl, C. Gabella, A. Bornert, I. F. Sbalzarini, J.-J. Meister, and A. B. Verkhovsky, Nat. Phys. 12, 367 (2016). 